\title{
Triggered/sequential star formation? A multi-phase ISM study around the prominent IRDC G18.93-0.03
}

\author{
J. Tackenberg ${ }^{1}$, H. Beuther ${ }^{1}$, R. Plume ${ }^{2}$, T. Henning ${ }^{1}$, J. Stil $^{2}$, M. Walmsley ${ }^{3}$, F. Schuller ${ }^{4}$, and A. Schmiedeke ${ }^{5}$ \\ ${ }^{1}$ Max-Planck-Institut für Astronomie (MPIA), Königstuhl 17, 69117 Heidelberg, Germany \\ e-mail: last-name@mpia.de \\ ${ }^{2}$ Department of Physics \& Astronomy and the Institute for Space Imaging Science, University of Calgary, Calgary, AB T2N 1N4, \\ Canada \\ 3 INAF - Osservatorio di Arcetri, Largo E. Fermi 5, 50125 Firenze, Italia \\ ${ }^{4}$ European Southern Observatory, Alonso de Cordova 3107, Vitacura, Santiago, Chile \\ 5 I. Physikalisches Institut der Universität zu Köln, Zülpicher Strasse 77, 50937 Köln, Germany
}

Received 31 July 2012 / Accepted 10 December 2012

\section{ABSTRACT}

\begin{abstract}
Context. Triggered star formation has been discussed for many years, and evidence for the formation of stars and cores triggered by HII regions is under debate.

Aims. We investigate the imprints of an expanding HII region on a pre-existing starless clumps.

Methods. We selected an infrared dark filament spanning $0.8^{\circ}$. One portion of this filament, G18.93-0.03 is a prominent dust complex, with the molecular clump G18.93/m being IR dark from near-IR wavelength up to $160 \mu \mathrm{m}$. Spitzer composite images show an IR bubble spatially associated with G18.93-0.03. We use GRS ${ }^{13} \mathrm{CO}$ and IRAM $30 \mathrm{~m} \mathrm{H}^{13} \mathrm{CO}^{+}$data to disentangle the large and small scale spatial structure of the region. From ATLASGAL submm data we calculate the gas mass, while we use the $\mathrm{H}^{13} \mathrm{CO}^{+}$line width to estimate its virial mass. To study the IR properties of G18.93/m we use Herschel data and produce temperature maps from fitting the spectral energy distribution. With the MAGPIS $20 \mathrm{~cm}$ and SuperCOSMOS $\mathrm{H}_{\alpha}$ data we trace the ionized gas, and the VGPS HI survey provides information on the atomic hydrogen gas.

Results. We show that the bubble is spatially associated with G18.93, located at a kinematic near distance of $3.6 \mathrm{kpc}$. The total gas mass of $\sim 870 M_{\odot}$ splits up into 6 sub-clumps, of which G18.93/m is the most massive with $280 M_{\odot}$. The virial analysis shows that it may be gravitationally bound and has neither Spitzer young stellar objects nor mid-IR point sources within. Therefore we call it pre-stellar. Fitting the spectral energy distribution reveals a temperature distribution that decreases towards its center, but heating from the ionizing source puts it above the general ISM temperature. We find that the bubble is filled by HII gas, ionized by an O8.5 star. Between the ionizing source and the IR dark clump G18.93/m we find a layered structure of hydrogen phases, from ionized to atomic to molecular gas, revealing a photon dominated region. Furthermore, we identify an additional velocity component within the bubble's $8 \mu \mathrm{m}$ emission rim at the edge of the infrared dark cloud and speculate that it might be shock induced by the expanding HII region. Conclusions. While the elevated temperature allows for the build-up of larger fragments, and the shock induced velocity component may lead to additional turbulent support, the density profile of G18.93/m does not show signatures of the expanding bubble. While the first two signatures favor high-mass star formation, we do not find conclusive evidence that the massive clump G18.93/m is prone to collapse because of the expanding HII region.
\end{abstract}

Key words. ISM: bubbles - stars: formation - stars: massive - HII regions

\section{Introduction}

Based on the Galactic plane survey GLIMPSE (Benjamin et al. 2003), it has become possible to study bubble-like structures in the Milky Way in a statistical sense. Initially, Churchwell et al. (2006) found a "bubbling Galactic disk" and identified 322 bubbles. Today, several compilations of bubbles in the Milky Way exist (e.g. Churchwell et al. 2006, 2007). The most extensive of which has been compiled by "The Milky Way Project" (Simpson et al. 2012), a citizen science project. They visually identified and classified more than 5000 bubbles. Infrared bubbles are defined by their bright $8 \mu \mathrm{m}$ emission rims. Although their origin may be manifold, many of the bubbles have shown to be created by a, not necessarily central, ionizing source. In this context, the emission rim originates from UV excited polycyclic aromatic hydrocarbons (PAHs). While all hydrogen ionizing photons are absorbed within the borders of an ionization front, lower energy
UV photons pass through the ionization front and may excite the PAHs. In addition, the ionizing source heats its surrounding gas, which expands and drives a shock front beyond the ionization front. In between the shock and ionization front, neutral material can be accumulated. Elmegreen \& Lada (1977) discussed the triggering of star formation on the borders of expanding HII regions. Among others, "collect and collapse" describes the collapse of swept up material along a compressed layer. Deharveng et al. (2003) and Zavagno et al. (2006) established "collect and collapse" observationally. A brief overview on triggered star formation is given in Deharveng et al. (2010).

Other results of the GLIMPSE survey are systematical and sensitive studies of infrared dark clouds (IRDCs, e.g. Peretto \& Fuller 2009). Previously discovered by the two infrared satellites ISO and MSX, their high column densities absorb the background emission even at infrared wavelengths and thus become visible as dark patches. With such high column densities, IRDCs 


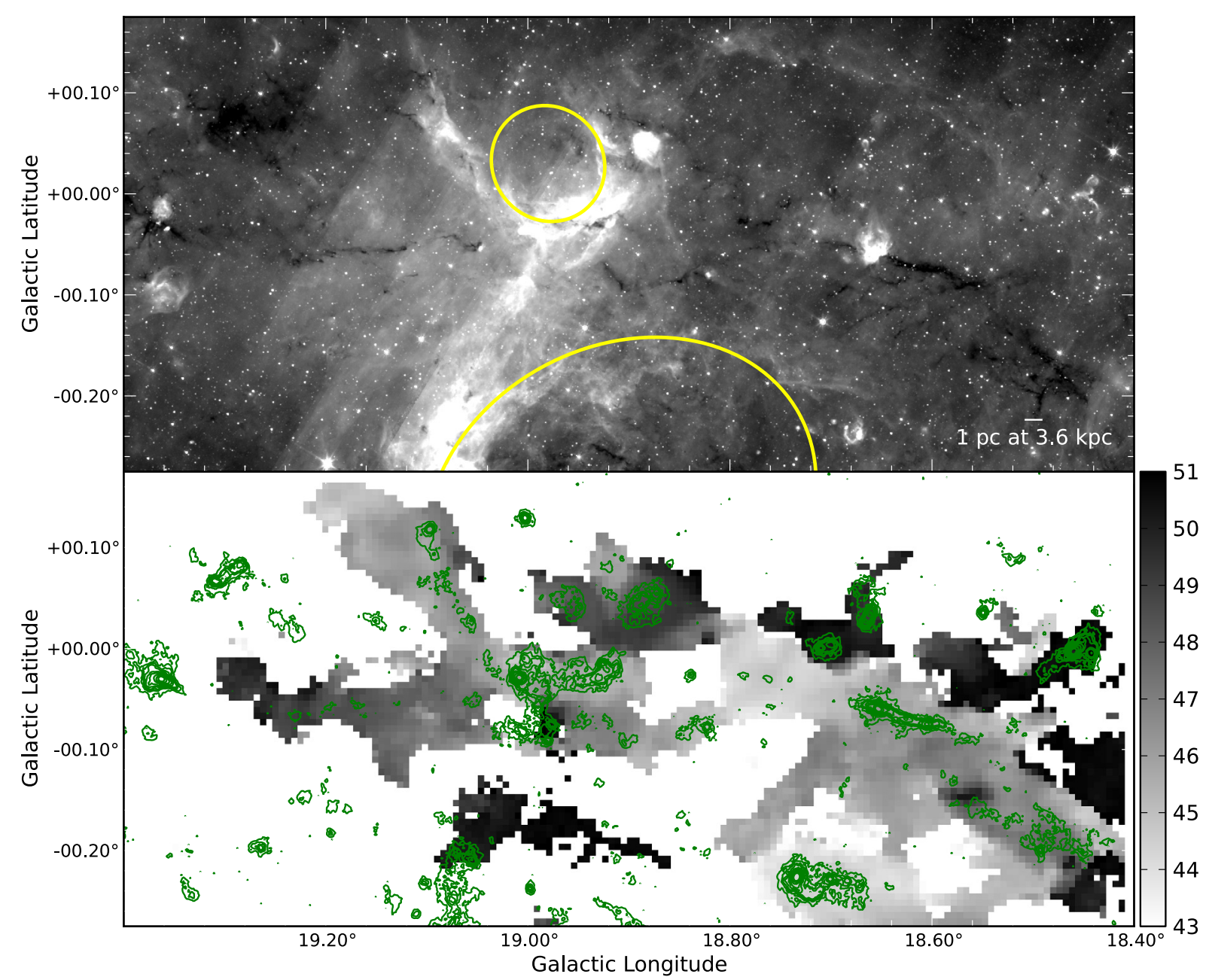

Fig. 1. An IRDC filament along the Galactic plane, spanning across from the lower right $\left(18.45^{\circ},-0.20^{\circ}\right)$ to the upper left $\left(19.30^{\circ},-0.02^{\circ}\right)$. The top panel shows a $8 \mu \mathrm{m}$ Spitzer image and the color stretch is chosen to bring the IRDC to prominence. The lower panel presents the intensity weighted peak velocity (moment 1) map of the GRS ${ }^{13} \mathrm{CO}$ data. Regions with velocities outside the $43 \mathrm{~km} \mathrm{~s}^{-1}$ to $51 \mathrm{~km} \mathrm{~s}^{-1}$ are clipped. The green contours are from ATLASGAL with contour levels at $0.15 \mathrm{Jy}$ or $3 \sigma, 0.3 \mathrm{Jy}, 0.4 \mathrm{Jy}, 0.5 \mathrm{Jy}, 0.7 \mathrm{Jy}, 0.9 \mathrm{Jy}, 1.3 \mathrm{Jy}, 1.8 \mathrm{Jy}$, and $2.5 \mathrm{Jy}$. The yellow ellipses indicate the bubbles as given in Simpson et al. (2012).

are believed to be the cradles of the next generation of stars (Rathborne et al. 2006; Simon et al. 2006). In addition, a rising number of large scale Galactic plane surveys allow one to trace the various stages of star formation. From mm and sub-mm observations of cold, dense clumps and cores (Schuller et al. 2009; Bally et al. 2010) to mid- to near-IR observations of young stellar objects (Benjamin et al. 2003; Carey et al. 2009; Molinari et al. 2010) and stars as well as the ionized and molecular gas (Condon et al. 1998; Jackson et al. 2006; Purcell \& Hoare 2010), an almost complete picture can be obtained for regions within the Galactic plane.

However, although both filamentary structures and IRDCs have gotten much interest over the last years, extreme IRDCs like the 80 pc long "Nessie" (Jackson et al. 2010) are still rare objects. A similar large scale filament of more than $50 \mathrm{pc}$ length has been presented by Kainulainen et al. (2011). Figure 1 shows this filament, spanning over $\sim 0.87^{\circ}$. At a kinematic near distance of $\sim 3.6_{-0.5}^{+1.0} \mathrm{kpc}$, this translates to a length of $\sim 54 \mathrm{pc}$. (For a discussion of the distance, see Sect. 3.2.) Its coherent velocity structure is shown in the bottom panel of Fig. 1. Following the low density gas traced by ${ }^{13} \mathrm{CO}$ along the extinction patch, the smooth velocity transitions suggest that the entire region is spatially connected. Kainulainen et al. (2011) studied the extinction map of the region and estimate the total gas reservoir to be $4.7 \times 10^{4} M_{\odot}$. Along the filament at lower longitudes, IRDC 18223 is a well-studied prototype IRDC with ongoing high-mass star formation (e.g. Beuther et al. 2010, and references therein).

A particularly interesting region in the context of triggered star formation is the prominent IRDC G18.93. Being part of the large scale filament described above, its location is also coincident with the rim of a IR bubble just above the filament (indicated by the upper yellow ellipse in Fig. 1). We will show that its dense gas, the filament and the bubble have coherent velocities and therefore are all at the same distance. Using CLUMPFIND on $870 \mu \mathrm{m}$ dust continuum data we identify six sub-clumps. The most massive clump, denoted G18.93/m, is even infrared dark from near infrared wavelengths up to $160 \mu \mathrm{m}$.

For the particular case of G18.93/m we will address the following questions: do we see evidence that the bubble influences G18.93/m? Do the properties of G18.93/m differ from other high-mass starless clumps?

We will first introduce the data used for the analysis and their implications. In Sect. 3 we introduce G18.93 and the neighboring bubble, and supplement a more detailed description in Sect. 3.3 and onwards. Section 4 describes the signs of triggered 


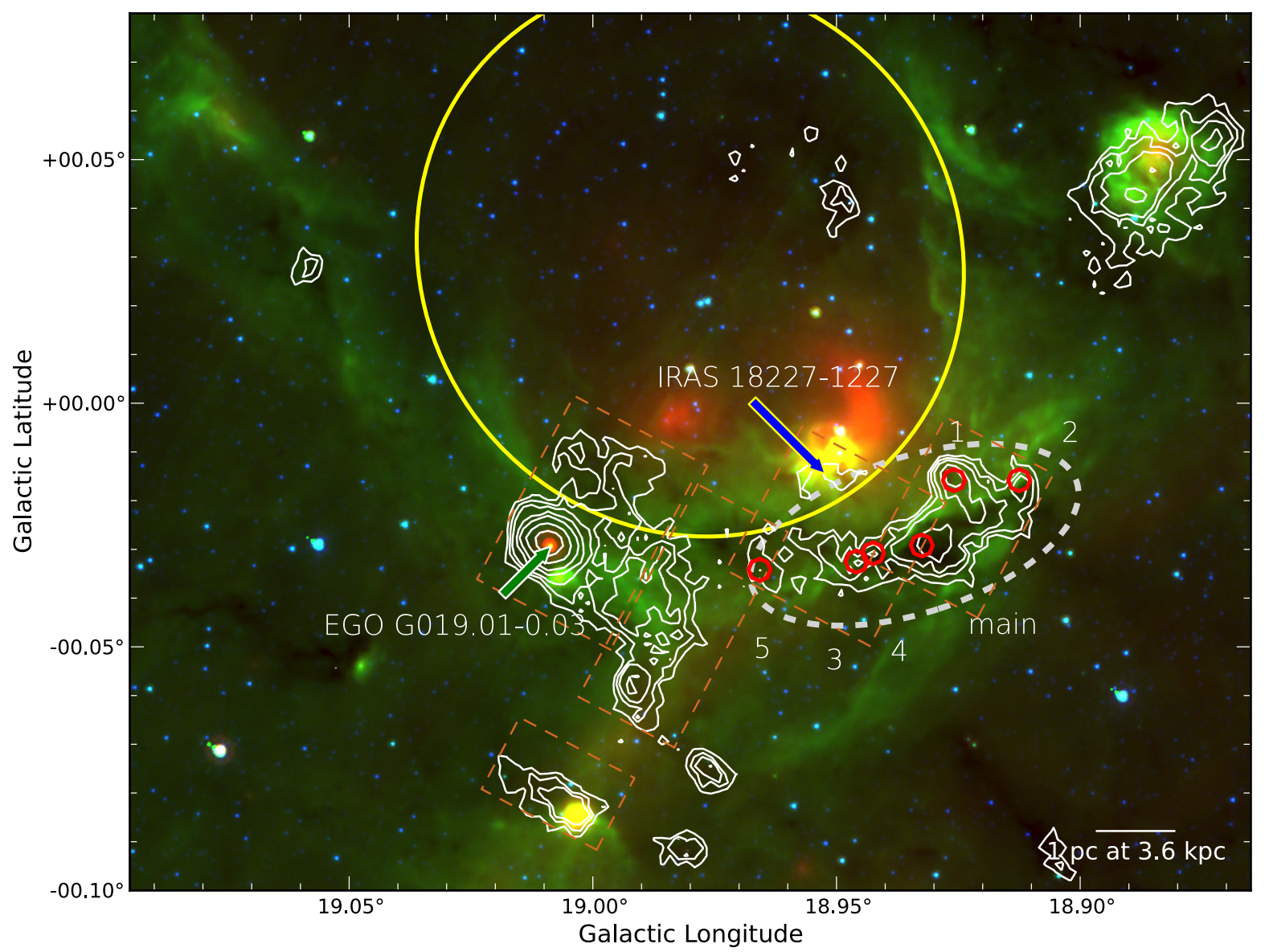

Fig. 2. Three color image of the bubble connected to the long filament, MWP1G018980+00304, composed from the Spitzer $3.6 \mu \mathrm{m}, 8 \mu \mathrm{m}$, and $24 \mu \mathrm{m}$ bands in blue, green, and red, respectively. White contours are from ATLASGAL (at 0.3 Jy, 0.4 Jy, 0.5 Jy, 0.7 Jy, 0.9 Jy, $1.3 \mathrm{Jy}, 1.8 \mathrm{Jy}$, and $2.5 \mathrm{Jy}$ ) for which the numbers denote the CLUMPFIND peaks, marked by the red circles. The yellow solid ellipse marks the bubble with interpolated bubble dimensions adopted from Simpson et al. (2012). The two arrows mark IRAS 18227-1227, and the massive proto-stellar object EGO G019.01-0.03. While the dashed orange boxes indicate the areas that have been mapped in $\mathrm{H}^{13} \mathrm{CO}^{+}$with the IRAM $30 \mathrm{~m}$ telescope, the gray dashed ellipse is drawn around the ATLASGAL contours connected to G18.93.

star formation we find and discusses their consequences. We summarize the paper in a concluding section.

\section{Description and implications of observations and data}

We used various publicly available surveys, as well as IRAM $30 \mathrm{~m}$ follow-up observations of the specific region.

\subsection{The cold dust tracing the molecular hydrogen, ATLASGAL}

Molecular hydrogen, $\mathrm{H}_{2}$, is hard to trace directly, and no pure rotational transitions are observable from the ground. However, in regions of high particle densities the gas thermally couples with the dust. Cold dust can be observed by its thermal radiation in the Rayleigh-Jeans regime at submm and mm wavelength. The APEX Telescope Large Area Survey of the Galaxy (ATLASGAL, Schuller et al. 2009) covers the full inner Galactic plane at $870 \mu \mathrm{m}$ with a resolution of $19.2^{\prime \prime}$ and an rms below $50 \mathrm{mJy}$. Using the Large APEX Bolometer CAmera (LABOCA, Siringo et al. 2009), each position of the inner Galactic plane has been mapped on-the-fly twice, each in different scanning directions. The pointing is on the order of 4 " and the calibration uncertainty is lower than $15 \%$. A detailed description of the data reduction is given in Schuller et al. (2009). The submm emission is mostly optically thin.

\subsection{IRAM 30 m observations}

We used the IRAM $30 \mathrm{~m}$ telescope to map the region around G18.93 identified in the ATLASGAL $870 \mu \mathrm{m}$ images (Schuller et al. 2009; Tackenberg et al. 2012) in $\mathrm{H}^{13} \mathrm{CO}^{+}$and $\mathrm{SiO}$.

$\mathrm{H}^{13} \mathrm{CO}^{+}$is a well established and common dense gas tracer with a critical density at $20 \mathrm{~K}$ of $\sim 1.8 \times 10^{5} \mathrm{~cm}^{-3}$. It is generally optically thin and yields information on the bulk motion of the gas (Vasyunina et al. 2011). SiO becomes released from the dust grains due to shocks and is therefore a well known tracer of shocked gas and molecular outflows (e.g. Schilke et al. 1997).

During our observing run in August 2010 the weather conditions were poor with average precipitable water vapour (pwv) $>10 \mathrm{~mm}$. Although the weather affected the pointing, it is, nonetheless, better than a third of the $3 \mathrm{~mm}$ beam (or $\sim 9^{\prime \prime}$ ). We used on-the-fly mapping on 5 boxes to cover all $870 \mu \mathrm{m}$ continuum emission above $0.3 \mathrm{Jy}$, or $2 \times 10^{22} \mathrm{~cm}^{-2}$ (assuming a general dust temperature of $15 \mathrm{~K}$, for further details see Sect. 3.3.1; see Fig. 2 for the coverage of the observations). The IRAM $30 \mathrm{~m}$ beam width for both $\mathrm{H}^{13} \mathrm{CO}^{+}$and $\mathrm{SiO}$ is $29.9^{\prime \prime}$ 
and the $3 \mathrm{~mm}$ setup chosen provides a spectral resolution of $40 \mathrm{kHz}$ at $40 \mathrm{MHz}$ bandwidth. This translates to a native velocity resolution of $0.14 \mathrm{~km} \mathrm{~s}^{-1}$ at $86 \mathrm{GHz}$. The data reduction was done using CLASS from the GILDAS ${ }^{1}$ package. We subtracted a linear baseline and removed the three central channels to avoid occasional spikes from the backends. The maps have been produced with a pixel scale of $14.2^{\prime \prime}$. With a total integration time of $11.3 \mathrm{~h}$ the average rms at a velocity resolution of $0.25 \mathrm{~km} \mathrm{~s}^{-1}$ is $0.08 \mathrm{~K}$ and $0.05 \mathrm{~K}$ for $\mathrm{H}^{13} \mathrm{CO}^{+}$and $\mathrm{SiO}$, respectively.

Simultaneously with the $3 \mathrm{~mm}$ observations, we recorded two of the $\mathrm{H}_{2} \mathrm{CO}(3-2)$ lines, $\mathrm{CH}_{3} \mathrm{OH}(4-3)$, and $\mathrm{HC}_{3} \mathrm{~N}(24-23)$ at $1 \mathrm{~mm}$. However, due to the poor weather conditions the average rms noise in the spectra is above $0.32 \mathrm{~K}$. With the given sensitivity, for the region of interest we do not detect any of the higher excitation lines at $1 \mathrm{~mm}$.

\subsection{The large scale cloud complex in HI, VGPS}

The ISM is composed primarily of atomic hydrogen. HI (self) absorption features are usually spatially correlated to molecular clouds and IRDCs (Li \& Goldsmith 2003). We used the $21 \mathrm{~cm}$ HI observations of the VLA Galactic Plane Survey (VGPS, Stil et al. 2006) to study the large scale distribution of atomic hydrogen. With the VLA in D configuration, the best resolution at $21 \mathrm{~cm}$ is $45^{\prime \prime}$. The interferometer data were supplemented with short spacings from the NRAO Greenbank telescope (GBT). To improve the survey's sensitivity to extended, low surface bright emission, the data were reduced to a synthesized beam of $1^{\prime}$ at a velocity resolution of $1.56 \mathrm{~km} \mathrm{~s}^{-1}$. The data cubes are sampled to a pixel scale of $18^{\prime \prime}$ at $0.8 \mathrm{~km} \mathrm{~s}^{-1}$. The calibration was made to be consistent with the NVSS survey (Condon et al. 1998).

\subsection{A large-scale view of the molecular cloud, GRS}

To study the molecular cloud component to its full extent we selected ${ }^{13} \mathrm{CO}$, a (well known) low density gas tracer with a critical density $\sim 1.9 \times 10^{3} \mathrm{~cm}^{-3}$. While it traces the same density range as ${ }^{12} \mathrm{CO}$, the isotopologue ${ }^{13} \mathrm{CO}$ has lower abundances and is therefore optically thinner. The Boston University-FCRAO Galactic Ring Survey (GRS, Jackson et al. 2006) provides spectroscopic ${ }^{13} \mathrm{CO}(1-0)$ observations of the Galactic plane for Galactic longitudes $18^{\circ} \leq l \leq 58^{\circ}$. The observations were performed with the single sideband focal plane receiver SEQUOIA, mounted on the Five College Radio Astronomy Observatory (FCRAO) $14 \mathrm{~m}$ telescope in OTF mode. Its angular resolution is $46^{\prime \prime}$, and the publicly available maps are sampled to a grid of $22^{\prime \prime}$. The velocity resolution is $0.2 \mathrm{~km} \mathrm{~s}^{-1}$, the pointing accuracy is better than $5^{\prime \prime}$.

\subsection{The stellar component of the complex, UKIDSS, GLIMPSE and MIPSGAL}

To examine the stellar content and stellar properties, we employed near- and mid-IR surveys. Both the Galactic Legacy Infrared Mid-Plane Survey Extraordinaire (GLIMPSE, Benjamin et al. 2003) and the MIPS Galactic plane survey (MIPSGAL, Carey et al. 2009) are Spitzer Legacy Programs. The GLIMPSE survey provides maps of the inner Galactic plane for $-60^{\circ} \leq l \leq 60^{\circ}$ at $3.8 \mu \mathrm{m}, 4.5 \mu \mathrm{m}, 5.8 \mu \mathrm{m}$, and $8.0 \mu \mathrm{m}$. The resolution is $1.7^{\prime \prime}, 1.7^{\prime \prime}, 1.9^{\prime \prime}, 2.0^{\prime \prime}$, respectively. From MIPSGAL we used the $24 \mu \mathrm{m}$ images with a resolution

\footnotetext{
1 http://wWw.iram.fr/IRAMFR/GILDAS
}

of $6 "$. Additional near-IR JHK data have been taken from the UKIDSSDR7PLUS data release (Lawrence et al. 2007).

\subsection{The peak of the SED, HiGal}

Covering the peak emission of the spectral energy distribution (SED) of cold dust, the HiGal/Herschel survey (Molinari et al. 2010) provides mid-IR observations at $70 \mu \mathrm{m}$ and $160 \mu \mathrm{m}$, and at submm wavelength $250 \mu \mathrm{m}, 350 \mu \mathrm{m}$, and $500 \mu \mathrm{m}$. Therefore, they connect the ATLASGAL submm observations of the cold dust at $870 \mu \mathrm{m}$ to the near- and mid-IR observations of the hot dust and stellar radiation. From a black body fit to the fluxes, we will estimate the temperature of the dust. The observations were carried out in parallel mode with a scanning speed of $60^{\prime \prime} / \mathrm{s}^{-1}$. Since the official, fully reduced HiGal fields are not yet available, we reduced the HiGal raw data using HIPE (level 0 to 1 , Ott 2010) and SCANAMORPHOS (level 1 to 2, v16.0, Roussel 2012). The angular resolutions at the given wavelengths are $10.2^{\prime \prime}, 13.6^{\prime \prime}, 23.4^{\prime \prime}, 30.3^{\prime \prime}$, and $42.5^{\prime \prime}$, respectively (Traficante et al. 2011).

\subsection{The ionized gas, MAGPIS and SHS}

In addition to thermal dust and line emission, free-free emission can be observed, which traces ionized gas. A certain amount of ionizing photons per second is required to maintain a given amount of gas in ionized state. This allows a characterization of the ionizing source. However, especially at $\mathrm{cm}$ wavelength another significant emission contribution is coming from the cosmic ray electrons' synchrotron radiation. Here, the spectral index can help to distinguish which contribution dominates.

The Multi-Array Galactic Plane Imaging Survey (MAGPIS, Helfand et al. 2006) mapped the $1.4 \mathrm{GHz}$, or $20 \mathrm{~cm}$, continuum of the first Galactic quadrant partially. Using the VLA in B, C, and $\mathrm{D}$ configurations allows a resolution of $\sim 6^{\prime \prime}$, while the extended structures are preserved by including Effelsberg $100 \mathrm{~m}$ data (Reich et al. 1990). The ready reduced maps have a pixel scale of $2^{\prime \prime} /$ pix.

While being very susceptible to extinction, the optical $\mathrm{H}_{\alpha}$ line traces the down cascade of an electron after recombining from ionized to atomic hydrogen. The SuperCOSMOS $\mathrm{H}_{\alpha}$ survey (Parker et al. 2005) digitized AAO/UKST narrow-band $\mathrm{H}_{\alpha}$ observations of the southern Galactic plane with a resolution of $\sim 1^{\prime \prime}$.

\section{Results}

The employment of the various surveys allows us to draw a comprehensive large scale picture of the cloud complex associated with the IRDC G18.93. In the following we will describe the environment, going from large to small scales. Finally we will use velocity information to study the spatial relations.

\subsection{A massive IRDC in the vicinity of a bubble}

As mentioned in the introduction, a prominent feature along the $>50 \mathrm{kpc}$ long filament is the bubble like structure indicated by the yellow ellipse in Fig. 1. A second bubble, N24 from Churchwell et al. (2006) and Deharveng et al. (2010) marked by the lower yellow ellipse in Fig. 1, is close in projection as well. However, while the filament has a velocity $\left(v_{\mathrm{lsr}}\right)$ around $\sim 45 \mathrm{~km} \mathrm{~s}^{-1}$, the second bubble has a distinct velocity at around $60 \mathrm{~km} \mathrm{~s}^{-1}$ and therefore is at a different distance. 


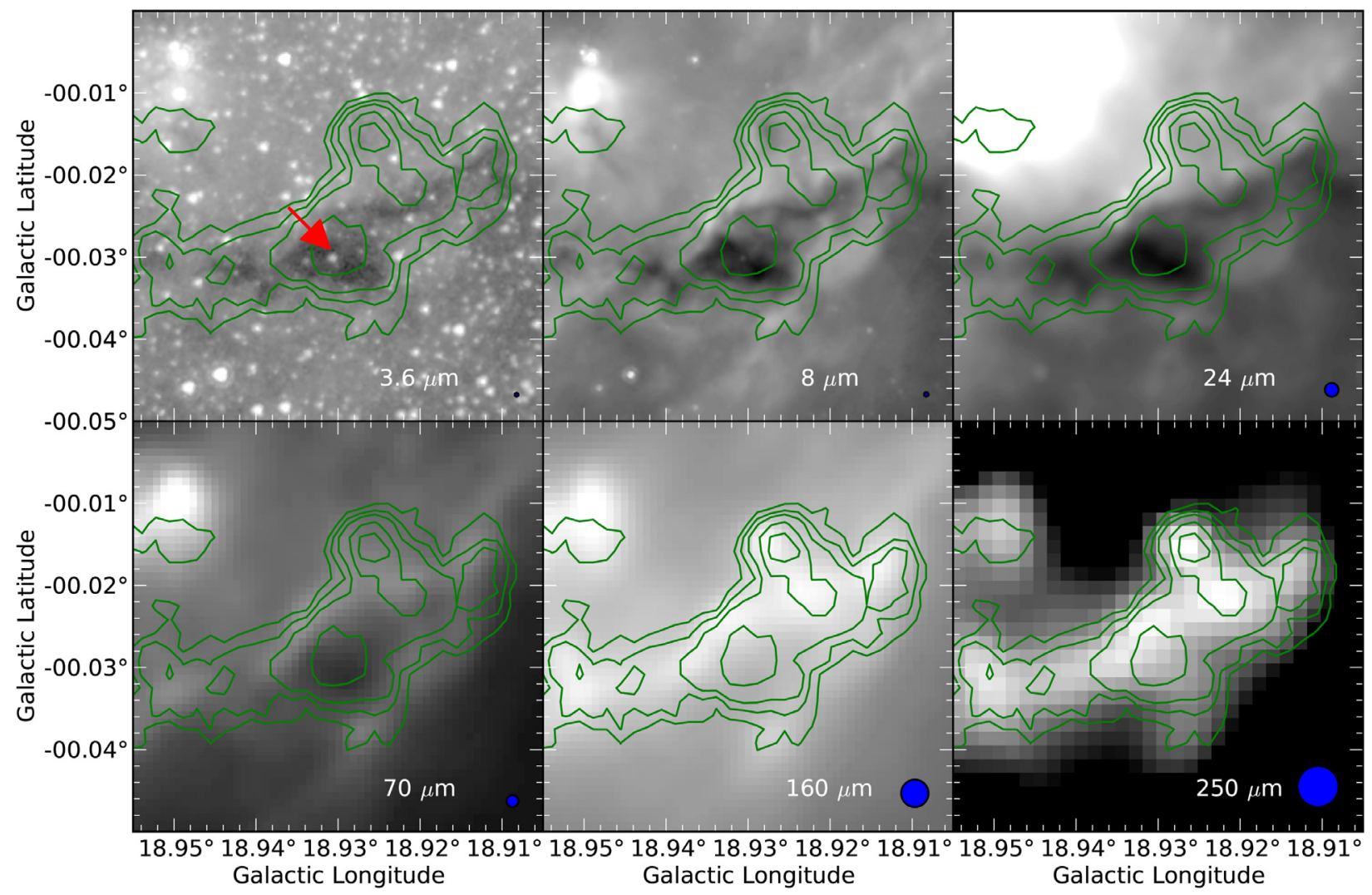

Fig. 3. A multi-wavelength zoom on G18.93/m, the continuum peak connected to the prominent IR extinction feature. The panels are (from left to right in reading order): $3.6 \mu \mathrm{m}, 8.0 \mu \mathrm{m}, 24 \mu \mathrm{m}, 70 \mu \mathrm{m}, 160 \mu \mathrm{m}, 250 \mu \mathrm{m}$, from GLIMPSE, MIPSGAL, and HIGAL. The blue circles in the bottom right of each panel indicate the beam sizes, the green contours are from ATLASGAL (at $0.3 \mathrm{Jy}, 0.4 \mathrm{Jy}, 0.5 \mathrm{Jy}, 0.7 \mathrm{Jy}, 0.9 \mathrm{Jy}, 1.3 \mathrm{Jy}, 1.8 \mathrm{Jy}$, and $2.5 \mathrm{Jy}$ ). The colors of the source marked by a red arrow in the first panel are not consistent with typical colors of young stellar objects (class $0 / 1$ ) (Gutermuth et al. 2008; Robitaille et al. 2008).

A zoom in on the smaller bubble closer to the filament is shown in Fig. 2. It opens towards higher Galactic latitudes and only little $24 \mu \mathrm{m}$ emission is visible within the bubble. The opening of the bubble resembles a typical "champagne flow" (Stahler \& Palla 2005) with lower density material at higher latitudes. On the lower inner edge of the rim a bright IRAS source is located together with two bright NIR sources.

Directly at the edge of the bubble at lower latitudes, several studies identified infrared dark clouds (IRDCs) and massive dense clumps, e.g. SDC G18.928-0.031 (Peretto \& Fuller 2009), [SMC2009] G18.93-0.01 (Schuller et al. 2009), or BGPS G18.926-0.019 (Rosolowsky et al. 2010). As shown in Fig. 2, the ATLASGAL survey at $870 \mu \mathrm{m}$ revealed several dense structures along the bubble. The largest structure in Fig. 2 is partly following the IRDC filament. In addition, Cyganowski et al. (2008) found an extended green object (EGO) within the rim of the bubble, EGO G19.01-0.03 which drives a bipolar outflow and is characterized as a genuine fast accreting massive young stellar object (Cyganowski et al. 2008, 2011a,b). As shown in Fig. 3, the main emission peak of the IRDC shows up in absorption up to even $160 \mu \mathrm{m}$. While absorption of IRDCs usually refers to extinction against the Galactic background from PAHs and very small grains, it is unclear whether the absorption feature at the longer wavelength of $160 \mu \mathrm{m}$ implies a lack of emission or simply no grains hot enough to emit in that line of sight. However, the part around EGO G019.01-0.03 and all connected continuum emission towards lower Galactic latitudes does not even show $24 \mu \mathrm{m}$ extinction signatures.

Within and along the bubble, there are few additional complexes dense enough to contain dense clumps and become visible at ATLASGAL sensitivity. However, since we want to study the influence of an expanding HII region upon an already existing filament, we will not discuss the continuum emission in the region that is clearly above the filament. Due to its characteristic absorption we will call the IR dark structure defined by the ATLASGAL emission G18.93 (see gray ellipse in Fig. 2). For reasons we will discuss in Sect. 3.2 we keep the ATLASGAL emission aligned with the Galactic latitude axis connected to EGO G019.01-0.03 separate.

It is interesting to note that both the massive young stellar object EGO G019.01-0.03 and the IRDC filament are at the interface of the bubbles shown in Fig. 1.

\subsection{Disentangling the spatial relations of $G 18.93$ and EGO G19.01}

From ${ }^{13} \mathrm{CO}$, partly shown in Fig. 1, it cannot be concluded whether the dense dust for the region of interest seen in ATLASGAL is part of the $45 \mathrm{~km} \mathrm{~s}^{-1}$ filament component or the $60 \mathrm{~km} \mathrm{~s}^{-1}$ component connected to the second bubble. To address that question and to disentangle the velocity structure of the dense molecular gas, we mapped G18.93 and the dust emission around EGO G19.01-0.03 in $\mathrm{H}^{13} \mathrm{CO}^{+}$, a dense gas tracer.

As shown in Fig. 4, the dense gas has two distinct velocity components. Consistent with earlier single spectra (Cyganowski et al. 2011b; Wienen et al. 2012), the IRDC G18.93 shows emission at velocities between $43 \mathrm{~km} \mathrm{~s}^{-1}$ and $47 \mathrm{~km} \mathrm{~s}^{-1}$, while the peak of EGO G19.01-0.03 and the ATLASGAL emission directly connected to it have velocities between $59 \mathrm{~km} \mathrm{~s}^{-1}$ and $63 \mathrm{~km} \mathrm{~s}^{-1}$. For both components, the dense molecular gas tracer 


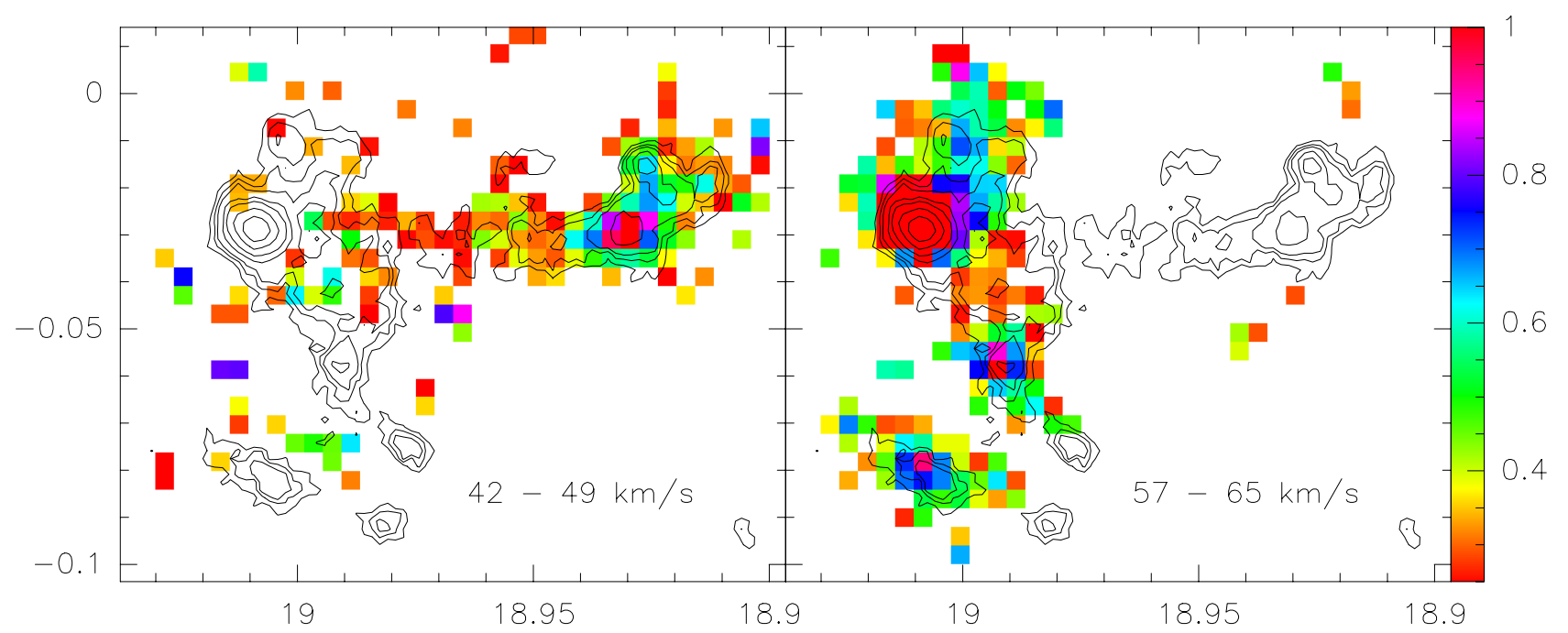

Fig. 4. Color coded map of the $\mathrm{H}^{13} \mathrm{CO}^{+}$emission in $\left[\mathrm{K} \mathrm{km} \mathrm{s}^{-1}\right.$ ] for the velocity regime $42 \mathrm{~km} \mathrm{~s}^{-1}$ to $49 \mathrm{~km} \mathrm{~s}^{-1}$ on the left, and $56 \mathrm{~km} \mathrm{~s}^{-1}$ to $65 \mathrm{~km} \mathrm{~s}^{-1}$ on the right. The black contours are from ATLASGAL with levels at $0.3 \mathrm{Jy}, 0.4 \mathrm{Jy}, 0.5 \mathrm{Jy}, 0.7 \mathrm{Jy}, 0.9 \mathrm{Jy}, 1.3 \mathrm{Jy}, 1.8 \mathrm{Jy}$, and $2.5 \mathrm{Jy}$.

resembles the structure defined by the ATLASGAL emission contours very well. Therefore, one can attribute the same velocities unambiguously to the ATLASGAL emission. That is not obvious from the lower density ${ }^{13} \mathrm{CO}$ alone. In addition, it is obvious that G18.93 is part of the long filament and the primary bubble which is connected to it, while EGO G19.01-0.03, a site of massive star formation and its connected material below this region, is at velocities consistent with the larger bubble from Churchwell et al. (2006).

In order to constrain the distance to the filament and the connected bubble, we employ the Galactic rotation curve given in Reid et al. (2009). Assuming a common velocity of $45 \mathrm{~km} \mathrm{~s}^{-1}$, the kinematic near distance becomes $3.6 \mathrm{kpc}$. From both extinction (Kainulainen et al. 2011) and considerations of the HI absorption feature (see Sect. 3.5 for details) we can conclude that the near distance is appropriate. Note that Kainulainen et al. (2011) give a kinematic distance of $4.1 \mathrm{kpc}$ and even larger distances from velocity independent methods. Therefore, error propagation resulting in a distance uncertainty of $0.05 \mathrm{kpc}$ underestimates the uncertainty. We expect the uncertainty rather to be on the order of $0.5 \mathrm{kpc}$.

\subsection{Details and aspects of the G18.93 complex}

So far we have shown that there is a large filament along the Galactic plane that has a consistent velocity structure over more than $50 \mathrm{pc}$. At $l=18.93^{\circ}, b=0.03^{\circ}$ the filament becomes very opaque, shows high mid-IR extinction and large gas column densities. Part of it shows IR absorption up to $160 \mu \mathrm{m}$. Just above G18.93, a bubble is visible. Its PAH rim visible at $8 \mu \mathrm{m}$ has a direct interface with G18.93. We will show later that the bubble is an expanding HII region. Therefore, G18.93 is an ideal source in which to study the influence of massive stars on starless clumps and search for imprints of triggering.

\subsubsection{The dust component of $\mathrm{G} 18.93$}

As mentioned in Sect. 3.2 and shown in Fig. 4, the densest gas (and most of the molecular hydrogen) in the complex is concentrated in the regions seen by ATLASGAL at $870 \mu \mathrm{m}$ (see Sect. 2.1).
In order to identify column density peak positions and associate masses to individual clumps, we used the CLUMPFIND algorithm of Williams et al. (1994). Starting at a $6 \sigma$ contour of $0.3 \mathrm{Jy}$, we chose $0.4,0.5,0.7,0.9,1.3,1.8$, and $2.5 \mathrm{Jy}$ as extraction thresholds. (For a detailed discussion of the extraction thresholds see Tackenberg et al. 2012.) The positions of the extracted clumps are listed in Table 1 and plotted in Fig. 2. We denote the prominent absorption dip and most massive clump G18.93/m.

Assuming optically thin thermal emission, the $\mathrm{H}_{2}$ gas column density can be calculated from the dust continuum emission via

$N_{\text {gas }}=\frac{R F_{\lambda}}{B_{\lambda}(\lambda, T) m_{\mathrm{H}_{2}} K \Omega}$,

with the gas-to-dust ratio $R=100, F_{\lambda}$ the flux at the given wavelength, $B_{\lambda}(\lambda, T)$ the blackbody radiation as a function of wavelength and temperature, $m_{\mathrm{H}_{2}}$ the mass of a hydrogen molecule, and the beam size $\Omega$. Assuming typical beam averaged volume densities in dense clumps of $10^{5} \mathrm{~cm}^{-3}$ and dust grains with thin ice mantles, the dust mass absorption coefficient from Ossenkopf \& Henning (1994) becomes $K=1.42 \mathrm{~cm}^{2} \mathrm{~g}^{-1}$ at $870 \mu \mathrm{m}$. The temperatures are taken from the HiGal temperature map described in Sect. 3.3.2. Because of the sparse resolution, we adopted the temperature at each peak position. The peak column densities at the spatial resolution of ATLASGAL (19.2") are then between $1.0 \times 10^{22} \mathrm{~cm}^{-2}$ to $4.2 \times 10^{22} \mathrm{~cm}^{-2}$.

With the distance as additional parameter and the integrated flux of the clump, the mass can be calculated in a similar way as given above,

$M_{\mathrm{gas}}=\frac{R d^{2} F_{\lambda, \mathrm{tot}}}{B_{\lambda}(\lambda, T) K}$

Assuming the kinematic distance of $3.6 \mathrm{kpc}$, the total mass becomes $\sim 870 M_{\odot}$, with clump masses between $\sim 30 M_{\odot}$ and $250 M_{\odot}$. Individual column densities and masses are listed in Table 1 . The given radius is the effective radius for equating the pixel area of each clump with a theoretical circular area, as calculated by CLUMPFIND. 
Table 1. CLUMPFIND decomposition of dust clumps extracted on ATLASGAL $870 \mu$ m map.

\begin{tabular}{|c|c|c|c|c|c|c|c|c|c|}
\hline Sub-clump name & $\begin{array}{c}\text { Gal. lon. } \\
{\left[{ }^{\circ}\right]}\end{array}$ & $\begin{array}{c}\text { Gal. lat. } \\
{\left[{ }^{\circ}\right]}\end{array}$ & $\begin{array}{c}\text { RA(2000) } \\
\text { [hh:mm:ss.s] }\end{array}$ & $\begin{array}{l}\operatorname{Dec}(2000) \\
\text { [dd:mm:ss] }\end{array}$ & $\begin{array}{l}\text { HiGal peak temp } \\
{[\mathrm{K}]}\end{array}$ & $\begin{array}{c}\text { Peak column density } \\
{\left[10^{22} \mathrm{~cm}^{-2}\right]}\end{array}$ & $\begin{array}{l}\text { Mass } \\
{\left[M_{\odot}\right]}\end{array}$ & $\begin{array}{c}\text { Angular radius } \\
{\left[{ }^{\prime \prime}\right]}\end{array}$ & $\begin{array}{c}\text { Radius } \\
{[\mathrm{pc}]}\end{array}$ \\
\hline 1 & 18.9259 & -0.0158 & $18: 25: 32.4$ & $-12: 26: 47$ & 23.0 & 4.2 & 248 & 27 & 0.46 \\
\hline main & 18.9325 & -0.0292 & $18: 25: 36.0$ & $-12: 26: 48$ & 21.7 & 3.5 & 276 & 29 & 0.50 \\
\hline 2 & 18.9125 & -0.0158 & $18: 25: 30.9$ & $-12: 27: 29$ & 22.3 & 2.4 & 104 & 21 & 0.36 \\
\hline 3 & 18.9459 & -0.0325 & $18: 25: 38.3$ & $-12: 26: 11$ & 22.5 & 2.2 & 107 & 22 & 0.38 \\
\hline 4 & 18.9425 & -0.0308 & $18: 25: 37.6$ & $-12: 26: 19$ & 22.4 & 2.1 & 57 & 15 & 0.27 \\
\hline 5 & 18.9658 & -0.0342 & $18: 25: 41.0$ & $-12: 25: 10$ & 23.0 & 1.5 & 43 & 15 & 0.26 \\
\hline
\end{tabular}

\subsubsection{Temperature map of $\mathrm{G} 18.93$}

The dust temperature is not only a necessary quantity for calculating column density and mass estimates, but it is an important physical parameter. As described in Sect. 2.6, we can use the HiGal/Herschel survey together with ATLASGAL data to estimate the dust temperature. Smoothing all data to the same resolution, we used frequency-dependent, optically thin dust emission models from Ossenkopf \& Henning (1994) to fit every pixel with a single temperature Planck function. (See also Ragan et al. 2012.) A common problem in the context of Herschel PACS/SPIRE data is the unknown background contribution. Several efforts have been made to solve that problem, e.g. Stutz et al. (2010); Battersby et al. (2011). In the context of determining the temperature we tested different background levels based on Gaussian fits to the noise distribution in regions with no or little signal. While the absolute temperatures differ up to $10 \%$, the relative temperature distribution is very similar. Therefore we refrain from subtracting any background and focus on the relative changes.

Furthermore, we have compared temperature maps using both HiGal and ATLASGAL, smoothed to a common resolution of $37^{\prime \prime}$, to temperature maps with only three bands, $70 \mu \mathrm{m}$, $160 \mu \mathrm{m}$, and $250 \mu \mathrm{m}$ at a resolution of $19^{\prime \prime}$. Although it is ambitious to fit a curve to only three data points, the peak of the SED is covered and can be reconstructed. The ATLASGAL data has been omitted since the missing background subtraction implies problems with the calibration and worsens the reconstruction of the peak. Comparing the temperatures of both SED fits on regions where differences due to the beam size are negligible, we find very good agreements. We want to point out that omitting the longer wavelength data biases the absolute results towards higher temperatures. However, the relative distribution in the temperature maps are preserved. Therefore, Fig. 5 shows the temperature maps at the better resolution of $19^{\prime \prime}$. To have more reliable absolute temperatures for deriving column densities and masses, we use the temperatures derived on all HiGal plus the ATLASGAL data.

As displayed in the top panel of Fig. 5, the dust around the IRAS source is hottest and produces a large scale temperature gradient. Nevertheless, all ATLASGAL peaks are colder than their neighborhood, and the temperature dip towards G18.93/m is the largest.

\subsubsection{G18.93/m: a starless clump}

Despite its prominence as absorption feature at Spitzer wavelengths, Tackenberg et al. (2012) did not list G18.93/m as a starless clump. Because of a peak in the extended $24 \mu \mathrm{m}$ emission within the clump boundaries, the clump had been rejected for consistency. In this detailed study however, we refrain from such rigorous methods and allow an individual inspection of the
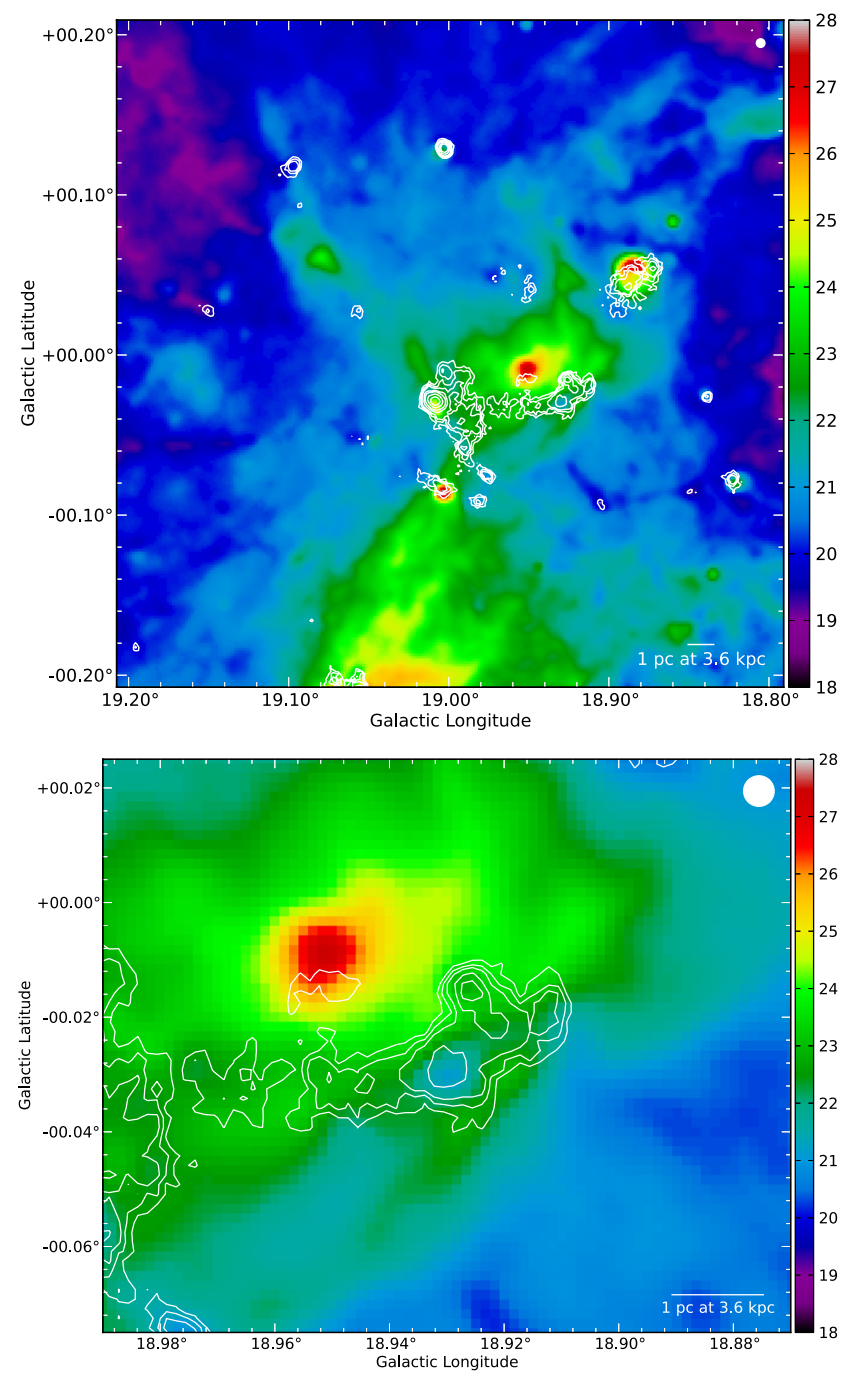

Fig. 5. Color coded temperature maps in $[\mathrm{K}]$ of G18.93, shown by the white contours from ATLASGAL (at $0.3 \mathrm{Jy}, 0.4 \mathrm{Jy}, 0.5 \mathrm{Jy}, 0.7 \mathrm{Jy}, 0.9 \mathrm{Jy}$, $1.3 \mathrm{Jy}, 1.8 \mathrm{Jy}$, and $2.5 \mathrm{Jy}$ ). The beam size is indicated in the top right corner. While the top panel presents the large-scale environment, the bottom panel zooms into our central IRDC region.

clump. Using the GLIMPSE color criteria given in Gutermuth et al. (2008), none of the cataloged near- and mid-IR sources within the clump is classified as young stellar object (class 0/I). Using similar GLIMPSE color criteria, Robitaille et al. (2008) did not identify young stellar objects projected onto the clump either. For even younger sources not yet visible at near-IR wavelength, Fig. 3 shows that there is no point source longwards of $8 \mu \mathrm{m}$ close to the ATLASGAL peak of G18.93/m. To further quantify our sensitivity we estimated the luminosity of objects 
that still could be embedded in the dust. Using the same assumptions as explained in Sect. 3.3.2, we here fitted two blackbody functions to the point source detection limits of the $24 \mu \mathrm{m}$ and $70 \mu \mathrm{m}$ images of $2 \mathrm{mJy}$ for MIPSGAL (Carey et al. 2009) and $\sim 10 \mathrm{mJy}$ for the $70 \mu \mathrm{m} \mathrm{HiGal}$ field together with the flux estimates for the absorption peak of $\mathrm{G} 18.93 / \mathrm{m}$ at $160 \mu \mathrm{m}$ and $250 \mu \mathrm{m}, 52.0 \mathrm{Jy}$ and $45.1 \mathrm{Jy}$. The warmer component then has only $0.1 L_{\odot}$, corresponding to a low-mass star of $0.14 M_{\odot}$. This can be considered as an upper limit for any potential embedded and undetected source.

Additionally indicative of true starless clumps is the absence of $\mathrm{SiO}$ emission. Since $\mathrm{SiO}$ traces outflows it is commonly used to differentiate between starless clumps and star forming clumps (Motte et al. 2007; Russeil et al. 2010). For the entire G18.93 complex we find no $\mathrm{SiO}$ down to column densities of $\sim 8 \times 10^{11} \mathrm{~cm}^{-2}$.

In order to understand whether $\mathrm{G} 18.93 / \mathrm{m}$ is pre-stellar or only a transient object, we compare its virial mass to its dust mass. While pre-stellar clumps are gravitationally bound and therefore will eventually form stars in the future, transient clumps are not gravitationally bound. Within such objects, smaller (here unresolved) fragments can still collapse, but the clumps itself will drift apart. As we will discuss in Sect. 4.5, we find a double peaked $\mathrm{H}^{13} \mathrm{CO}^{+}$profile at the ATLASGAL peak position of G18.93/m. As shown in Fig. 11, the line width of the component we attribute to the IRDC is $\delta v=2.1 \mathrm{~km} \mathrm{~s}^{-1}$. (For details see Sect. 4.5). To calculate the virial mass we use the equation given in MacLaren et al. (1988), $M_{\mathrm{vir}}=k R \delta v^{2}$. For the source radius $R$ we use $0.5 \mathrm{pc}$ as given in Table 1 , and the geometrical parameter $k$ depends on the density structure of the clump and is $k=190$ for $\rho \propto 1 / \mathrm{r}, k=126$ for $\rho \propto 1 / \mathrm{r}^{2}$. The mass then becomes $M_{\text {vir }}=420 M_{\odot}$ for a geometrical parameter $k=190$ or $\rho \propto 1 / \mathrm{r}$, and $M_{\text {vir }}=280 M_{\odot}$ with $k=126$ or $\rho \propto 1 / \mathrm{r}^{2}$. Beuther et al. (2002) and Hatchell $\&$ van der Tak (2003) find typical density distributions in sites of massive star formation of $\rho \propto \mathrm{r}^{\alpha}$ with $\alpha \sim-1.6$, in between both parameters. Doing a linear interpolation, the suggested virial mass becomes $360 M_{\odot}$. Considering all uncertainties, a comparison to the estimated dust mass for $\mathrm{G} 18.93 / \mathrm{m}$ of $280 M_{\odot}$ does not allow a conclusive distinction whether clump G18.93/m is gravitationally bound or not. However, as discussed in Sect. 3.3.2 the dust temperatures derived from SED fitting to Herschel data are very uncertain due to missing background levels. Comparing gas temperatures measured with $\mathrm{NH}_{3}$ observations (Pillai et al. 2006) to dust temperatures from Herschel observations (Henning et al. 2010), we find that despite the efficient dust cooling the ammonia temperatures are usually lower. Thus, the given temperatures are only upper limits and the clump masses may be higher. Therefore, the virial analysis is consistent with G18.93/m being gravitationally bound, and pre-stellar.

\subsection{The bubble}

According to Simpson et al. (2012), the bubble connected to the filament and described in Sect. 3.1, or MWP1G018980+000304, has an effective radius of 3.44'. However, as mentioned before, its appearance is more similar to a "champagne flow" (cf. Fig. 2). Since the expansion velocity of an HII region depends on the surrounding density, an inhomogeneous medium leads to asymmetric bubbles. If the ionizing gas reaches the edge of its parent molecular cloud or encounters dense gas, it will fan out towards the empty space (Stahler \& Palla 2005). Therefore, the effective radius and the major- and minor-axis can describe the current appearance of a bubble, but cannot describe the expansion history.

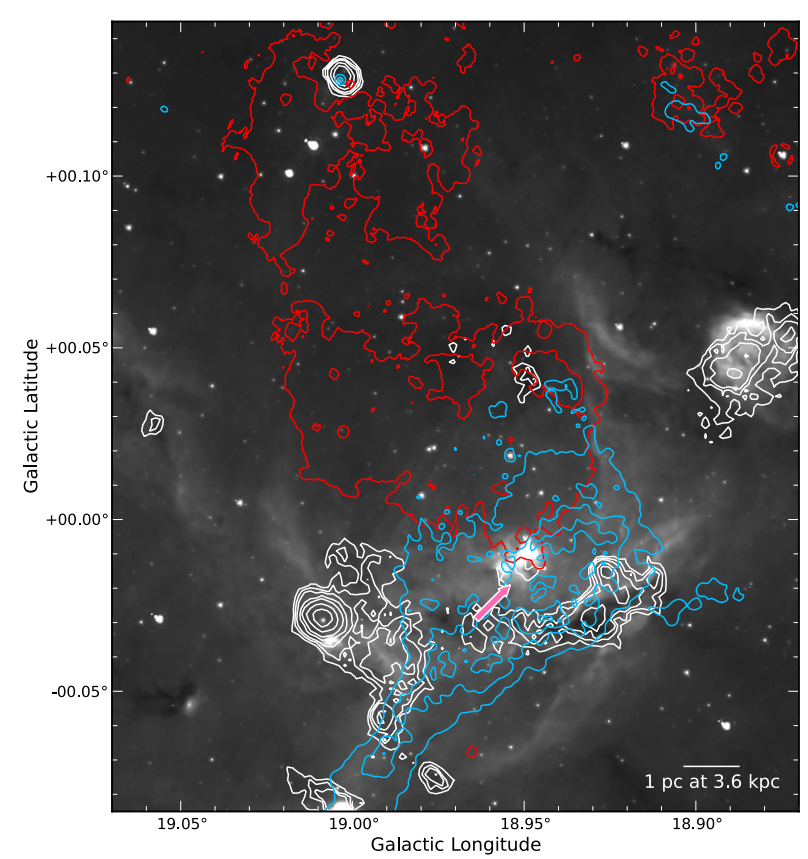

Fig. 6. The ionized gas within and around the bubble. On top of the GLIMPSE $8 \mu \mathrm{m}$ image, the red contours show the $\mathrm{H}_{\alpha}$ emission from the SuperCOSMOS survey, the blue contours represent the MAGPIS $20 \mathrm{~cm}$ emission, and white contours show the ATLASGAL emission (at $0.3 \mathrm{Jy}, 0.4 \mathrm{Jy}, 0.5 \mathrm{Jy}, 0.7 \mathrm{Jy}, 0.9 \mathrm{Jy}, 1.3 \mathrm{Jy}, 1.8 \mathrm{Jy}$, and $2.5 \mathrm{Jy}$ ). The contour levels for the MAGPIS data are $0.002 \mathrm{Jy} /$ beam, $0.003 \mathrm{Jy} / \mathrm{beam}$, $0.004 \mathrm{Jy} / \mathrm{beam}$, and $0.005 \mathrm{Jy} / \mathrm{beam}$. The arrow indicates the peak position of the HII region identified by Lockman (1989).

Thus we have to ask: what is the origin of MWP1G018980+00304? Galactic bubbles are usually produced by OB stars or clusters, driving an expanding HII region, or by stellar winds and radiation pressure of late B-type stars (Elmegreen \& Lada 1977; Churchwell et al. 2006; Deharveng et al. 2010; Simpson et al. 2012). For both processes, the exciting source is not necessarily in the middle of the bubble.

Often, (ultra) compact HII regions (UCHIIs) are found connected to the bubble driving source, but e.g. CORNISH (Purcell et al. 2008) does not find an UCHII region inside the bubble. However, despite its high sensitivity at $6 \mathrm{~cm}$ of better than $2 \mathrm{~m} \mathrm{Jy}$, due to interferometry techniques, CORNISH is not sensitive to sources larger than 12" (Hoare et al. 2012). Searching for more extended structure, Lockman (1989) conducted a $\mathrm{H}_{\alpha}$ radio recombination line single dish survey at $3 \mathrm{~cm}$ and detected an HII region at $l=18.954, b=-0.019$. As shown in Fig. 6, this is right between the IRAS source and G18.93, but measured with a beam of $3^{\prime}$. Although they measure a source velocity of $v_{\text {LSR }}=(52.3 \pm 1.7) \mathrm{km} \mathrm{s}^{-1}$, we believe that the bubble and the HII gas are spatially connected. Indeed, velocity offsets between the ionized gas and CO are common (Blitz et al. 1982; Fich et al. 1982). For the same HII region Kuchar \& Clark (1997) measure the flux at $4.85 \mathrm{GHz}$ with a beam width of $4.2^{\prime}$. For an estimated source diameter of $7.0^{\prime}$ they find $2146 \mathrm{mJy}$. Using the formula given in Kurtz et al. (1994) and assuming a correction factor for the optical depth of $a=0.9922$ (corresponding to a frequency $v=5 \mathrm{GHz}$, and temperature $T=9000 \mathrm{~K}$ as given in Mezger \& Henderson 1967), this flux translates to the number of Lyman continuum photons $n_{\mathrm{Ly}},=\log \left(N_{\mathrm{Ly}}\right)=48.4 \mathrm{~s}^{-1}$.

The blue contours in Fig. 6 show the MAGPIS GPS data at $20 \mathrm{~cm}$. The emission peak of the high resolution data at $20 \mathrm{~cm}$ 
agrees well with the extrapolated peak position from Lockman (1989) in the sense that it also lies between the IRAS source and G18.93. However, the GPS data shows $20 \mathrm{~cm}$ emission towards the IRDC and beyond. The higher latitude flux within the contour level $3 \times 10^{-3} \mathrm{Jy} /$ beam, without the tail towards lower latitudes, becomes 1.5 Jy. With $a=0.9951$ at $9000 \mathrm{~K}$ (Mezger \& Henderson 1967), that converts to a Lyman continuum flux of $n_{\text {Ly }}=48.2 \mathrm{~s}^{-1}$. A slightly larger contour drawn along the visual extent of the emission provides a flux of $2.5 \mathrm{Jy}$, thus $2 / 3 \mathrm{rd}$ bigger. However, with $n_{\mathrm{Ly}}=48.4 \mathrm{~s}^{-1}$ the logarithm does not change as much and the given differences can be considered as uncertainty in our measurements. Therefore, the results of Kuchar \& Clark (1997) and our measurements agree within the uncertainties.

As mentioned in Sect. 2.7, the synchrotron radiation of cosmic ray electrons can also contribute to the $\mathrm{cm}$ continuum emission. However, the spectral index $\alpha$ (with $\mathrm{S}_{v} \propto v^{-\alpha}$ ) of the thermal free-free emission is negative in this regime, while the spectral index of the synchrotron radiation is positive. Therefore, we can use the two independent measurements of the continuum emission to at least determine the sign of the spectral index. The slope between the $20 \mathrm{~cm}$, or $1.5 \mathrm{GHz}$, data point and the $6 \mathrm{~cm}$, or $4.85 \mathrm{GHz}$, data point is positive, suggesting a negative spectral index, which is in agreement with the expected spectral index for thermal free-free emission. Therefore, the emission is dominated by free-free radiation and we can neglect any synchrotron contribution. Nevertheless, with only two data points we cannot distinguish whether the assumption of optically thin emission is correct. Therefore, the given Lyman continuum fluxes are lower limits.

While the $\mathrm{cm}$ continuum is more prominent towards the dust continuum emission, large parts of the bubble are filled by SuperCOSMOS $\mathrm{H}_{\alpha}$ emission, see Fig. 6. As mentioned in Sect. 2.7, $\mathrm{H}_{\alpha}$ is a complementary tracer of ionized gas. The difference in the spatial distribution can be explained by the optical depth. Since the optical $\mathrm{H}_{\alpha}$ line is susceptible to extinction, $\mathrm{H}_{\alpha}$ can only be detected in regions of low visual extinction. However, the near-IR extinction map shows that the visual extinction towards the ATLASGAL emission peaks are larger than $A_{\mathrm{V}}=25 \mathrm{mag}$.

Using the averaged number of Lyman photons, $n_{\mathrm{Ly}}=$ $48.3 \mathrm{~s}^{-1}$ to determine the spectral type, the exciting source needs to be at least a main sequence star of type O8.5 (Panagia 1973; Martins et al. 2005). The extended appearance (and the connected non-detection within CORNISH) and the morphology of the HII suggests that the ionizing source is not extremely young. Nevertheless, the diameter of the bubble does not allow for extremely old bubble-driving sources.

From the mid infrared data, a good candidate for the ionizing source is IRAS $18227-1227$, marked in Fig. 2. It is very bright at $24 \mu \mathrm{m}$, but by $3.6 \mu \mathrm{m}$ it is no longer the brightest source within its neighborhood. At higher latitudes, $\sim 16^{\prime \prime}$ above IRAS $18227-1227$, a near-IR source, hardly visible at $24 \mu \mathrm{m}$, is another potential candidate to drive the bubble. Although the additional source appears blue in the four IRAC bands from GLIMPSE, the color criteria given in Gutermuth et al. (2008) identify it as young stellar object. At the even shorter wavelengths of the 2MASS and UKIDSS near-IR JHK surveys, the second source has a bright neighbor. Nevertheless, its missing detection in the GLIMPSE survey and its blue appearance at NIR wavelength indicates that it is evolved. However, the near infrared colors do not allow a classification or mass determination. While for one its classification as young stellar object (class I) hints to non photospheric emission at near infrared wavelength, the second source shows a significant color excess in the $J H K$ color-color diagram indicating non photospheric emission as well. Therefore we can not identify the exact ionizing source with the current data. Nevertheless, as shown before from $\mathrm{cm}$ free-free emission, it has to be of spectral type 08.5 or earlier.

Another result one can determine from the different tracers of the ionized gas are the densities above and below the ionizing source. While we have material that is optically thick to visible light suggesting dense gas towards the dust continuum emission, we have optically thin low density material within the bubble and above it. That explains the position of the ionizing source and the "champagne flow".

\subsection{The photon-dominated region: a layered structure}

What is the observable impact of the HII region on the dense filament, described in Sect. 3?

During the evolution of an HII region the temperature difference between the hot ionized gas and the cold environment drives a supersonic shock front beyond the ionization front. In addition, at the interface between an HII region and the neighboring gas a photon dominated region builds up. While hydrogen ionizing radiation with energies above $13.6 \mathrm{eV}$ produces the HII, beyond the ionization front other molecules with lower ionization energies may still be ionized. However, connected to the photon dominated region one expects a layered structure of HII-HI-H ${ }_{2}$ (Hollenbach \& Tielens 1997). For our bubble we can directly observe the layers between the presumably ionizing source and the dense gas of G18.93/m.

While the ionized gas and its structure is directly traced by the $\mathrm{cm}$ free-free emission, the ATLASGAL emission connected to $\mathrm{G} 18.93 / \mathrm{m}$ represents the cold dust, which is embedded in dense $\mathrm{H}_{2}$. In order to trace the morphology of the atomic hydrogen we employ the VGPS HI survey.

(Hot) HI is so abundant in the Galaxy, that along the Galactic plane, background HI emission is present at all velocities. Therefore, cold sources in the foreground appear as absorption features superimposed on the background HI emission. However, since the HI distribution and temperature vary strongly within the Galactic disk, the observed background varies strongly as well. In addition, individual HI clouds often have sufficiently high column densities to become self absorbing. This complicates the identification of complexes in the Hi data.

However, as shown in Fig. 7, the absorption dip for the dense gas around G18.93 is very prominent. In order to study the distribution of the atomic hydrogen, we quantified the absorption feature by the following process: (1) we fit the background in the spectra with a third order polynomial omitting the velocity range from $40 \mathrm{~km} \mathrm{~s}^{-1}$ to $50 \mathrm{~km} \mathrm{~s}^{-1}$. This determines the large scale background variations, but preserves the signals on small scales; (2) we subtract the fitted function as a baseline; (3) we invert the spectra and fit the now emission peak with a Gaussian. The integrated area of the Gaussian for a constant temperature is proportional to the column density. (NOTE: we stress that the authors do not want to convert the so quantified measure of $\mathrm{HI}$ into physical meaningful units, but only take it as relative value). Doing this procedure on all HI spectra around G18.93/m, we obtain the qualitative distribution of the atomic hydrogen.

A problem with this method arises from the free-free continuum. Due to the additional background emission towards the HII region, the absorption dip becomes stronger for the same HI column densities. Therefore, the interpretation of the HI distribution needs to be considered with caution. However, since 


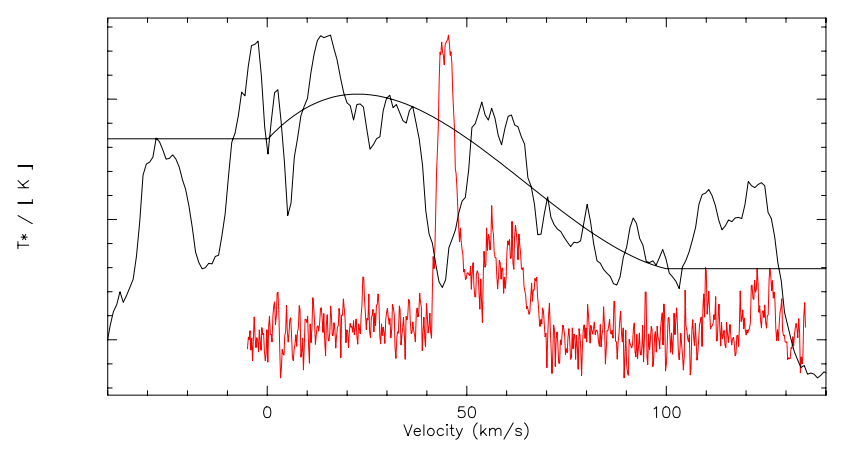

Fig. 7. HI VGPS spectrum of G18.93/m in black with the GRS ${ }^{13} \mathrm{CO}$ spectrum in red on top. The strong absorption feature of G18.93/m at $\sim 45 \mathrm{~km} \mathrm{~s}^{-1}$ in the HI spectrum agrees very well with the ${ }^{13} \mathrm{CO}$ signature. The smooth line indicates the 3rd order fit to the HI spectrum that has been used to subtract the "continuum". (For details see text.)

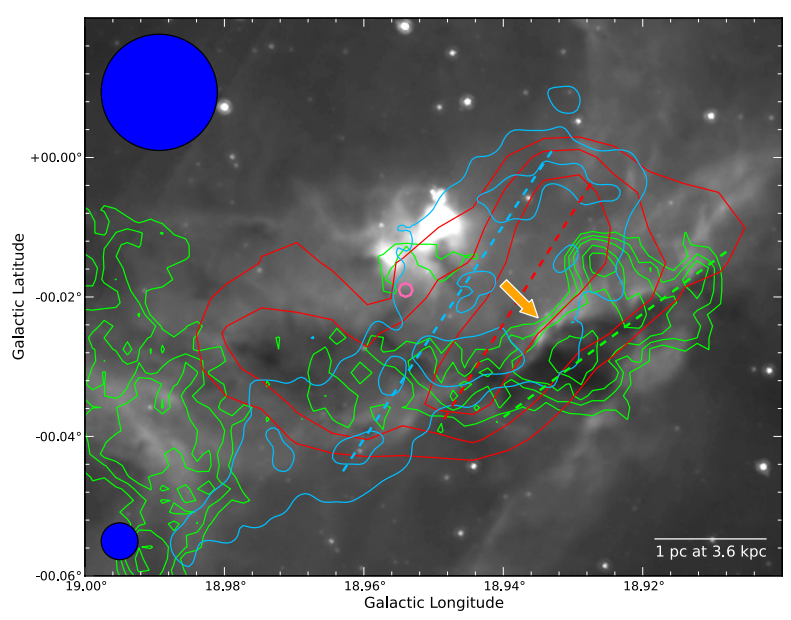

Fig. 8. A layered structure of the hydrogen phase. On top of the $8 \mu \mathrm{m}$ GLIMPSE image in gray, the blue contours represent the MAGPIS GPS $20 \mathrm{~cm}$ data, the red contours show a measure of the atomic hydrogen at velocities of G18.93 from the VGPS $21 \mathrm{~cm}$ line (for details see the text), and the green contours are the cold dust from the ATLASGAL survey, hence the molecular hydrogen. The dashed lines are to guide the eye and indicate the peak positions of the different data. The orange arrow points to $8 \mu \mathrm{m}$ excess emission at the edge of the dense gas. The top left circle indicates the beam size of the VGPS data, while the bottom circle shows the beam of ATLASGAL. In this figure, MAGPIS is smoothed to the resolution of ATLASGAL. The contour levels for the GPS $20 \mathrm{~cm}$ data are $2 \mathrm{mJy} /$ beam, $3 \mathrm{mJy} / \mathrm{beam}, 4 \mathrm{mJy} / \mathrm{beam}$, and $5 \mathrm{mJy} / \mathrm{beam}$, and for the ATLASGAL $870 \mu \mathrm{m}$ emission $0.3 \mathrm{Jy}, 0.4 \mathrm{Jy}, 0.5 \mathrm{Jy}, 0.7 \mathrm{Jy}, 0.9 \mathrm{Jy}$, $1.3 \mathrm{Jy}, 1.8 \mathrm{Jy}$, and $2.5 \mathrm{Jy}$.

the peak of the HI and HII emission do not agree, the suggested Hi distribution seems to be dominated by the Hi column density.

Figure 8 shows three physical states of hydrogen. Between the ionizing source and G18.93 a layered structure is visible, with first the ionized gas, then the atomic hydrogen, and then the molecular gas within the IRDC. However, one should keep in mind that the beam of the HI data is on the same order as the spacing between the different peaks.

\section{Imprints of triggering? Discussing the interaction between $\mathrm{G} 18.93 / \mathrm{m}$ and the expanding HII region}

So far, most studies of triggered star formation (e.g. Deharveng et al. 2003; Zavagno et al. 2006) have searched for young stellar objects connected to bubbles and HII regions. To understand the statistical significance of triggered star formation, current and future studies make use of the rising number of Galactic plane surveys (Zavagno et al. 2010; Deharveng et al. 2010; Kendrew et al. 2012). Although it has been shown in the past that not all stars on the border of HII regions are necessarily formed by triggering, it becomes clear that triggering might have a significant effect on star formation.

As a next step towards a better understanding of triggered star formation we need to observationally identify the mechanisms that govern the interaction between expanding HII regions and the molecular gas. In this context, detailed studies of individual regions are needed to reveal signatures of triggering before star formation sets in (e.g. Bieging et al. 2009).

In the following section we will explore whether we can see any imprints of the HII region on the starless clump G18.93/m.

\subsection{Comparison of $\mathrm{G18.93/m}$ with typical high-mass starless clumps}

As discussed in Sect. 3.3.3, G18.93/m is a starless clump with no IR emission up to $160 \mu \mathrm{m}$. While the virial analysis does not allow a firm conclusion as to whether it is bound or not, but if it is bound it would be a proto-type pre-stellar clump. In terms of mass and size it does not stand out. If we compare it to the starless clumps found in Tackenberg et al. (2012), its clump mass of $280 M_{\odot}$ is comparable to the average mass of $315 M_{\odot}$ they find. Note that with the lower dust opacity of $\kappa=0.77 \mathrm{~cm}^{2} \mathrm{~g}^{-1}$ used in Tackenberg et al. (2012) as well as the low temperature of $15 \mathrm{~K}$ they assume for starless clumps, the clump mass becomes $900 M_{\odot}$. Nevertheless, for peculiar sources such as $\mathrm{G} 18.93 / \mathrm{m}$, the elevated dust temperature needs to be taken into account.

If we assume a spherical clump we can calculate the average volume density of $\mathrm{G} 18.93 / \mathrm{m}$ to be $1 \times 10^{4} \mathrm{~cm}^{-3}$. This is significantly smaller than the $5.0 \times 10^{4} \mathrm{~cm}^{-3}$ for the sample of starless clumps presented in Tackenberg et al. (2012). However, from the detection of $\mathrm{H}^{13} \mathrm{CO}^{+}$with a critical density of $\sim 1.8 \times 10^{5} \mathrm{~cm}^{-3}$, we know that at least in the central regions the density needs to be higher. Nevertheless, since the average densities of the starless clumps have been calculated with the same assumptions, the results should be comparable. For the formation of high-mass stars, Krumholz \& McKee (2008) require a peak column density of $3 \times 10^{23} \mathrm{~cm}^{-2}$. With a peak column density of $4 \times 10^{22} \mathrm{~cm}^{-2}$, G18.93/m does not meet their requirements. However, the measured column densities are beam averaged values, true peak column densities are expected to be higher (Vasyunina et al. 2009). Although the expanding HII region might have influenced the starless clump G18.93/m, it does not differ from other starless clumps.

\subsection{The photon dominated region: a layered structure}

While the general distribution of the atomic hydrogen follows the ATLASGAL emission, the peak of HI is elongated towards the gap between the ionizing source and the molecular hydrogen, shown by Fig. 8. Towards G18.93/5 (cf. Fig. 2), the $20 \mathrm{~cm}$ freefree emission is no longer parallel to the cold dust, but crosses the IRDC. However, both extinction and dust emission are significantly lower at the intersection. That implies that there is less dense gas which allows the ionized gas to more easily escape beyond G18.93/5; the ionizing radiation is simply no longer blocked across the full height of the filament.

In the context of star formation, Glover \& Clark (2012) have shown that the composition of the gas has hardly any influence 

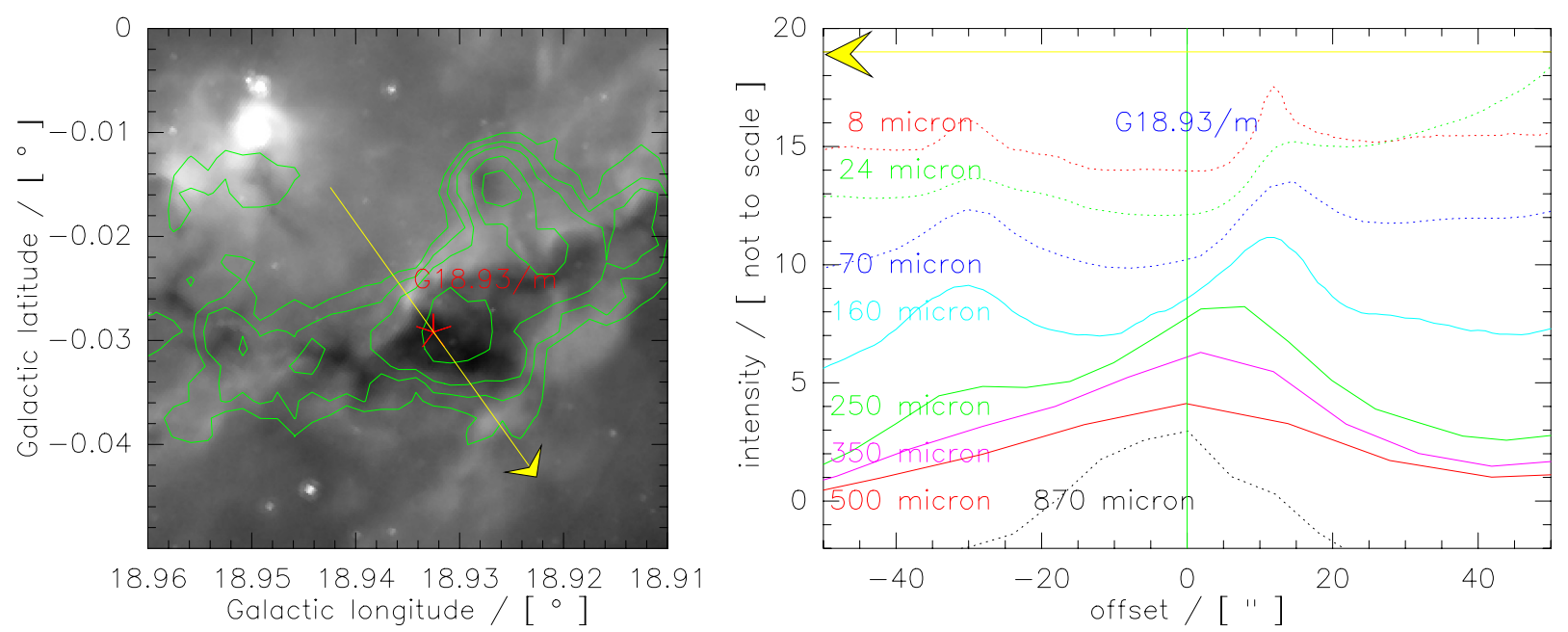

Fig. 9. Profiles of mid-IR data along the cut indicated by the yellow arrow in the left panel. While the center of the cut in the right panel is G18.93/m, its upper end is chosen to be towards the potential ionizing sources. The different colored profiles in the right panel are of increasing wavelength, starting with $8 \mu \mathrm{m}$ at the top going to $870 \mu \mathrm{m}$ at the bottom. The intensities are not to scale, but adopted to fit in the panel. The green vertical line indicates the position of the ATLASGAL continuum peak, the yellow arrow corresponds in direction and length to the arrow in the left panel, in which the background image is a GLIMPSE $8 \mu \mathrm{m}$ image with ATLASGAL contours (at $0.3 \mathrm{Jy}, 0.4 \mathrm{Jy}, 0.5 \mathrm{Jy}, 0.7 \mathrm{Jy}, 0.9 \mathrm{Jy}, 1.3 \mathrm{Jy}$, $1.8 \mathrm{Jy}$, and $2.5 \mathrm{Jy}$ ) on top.

on the star formation efficiency. They suggest that molecular gas is not a prerequisite for the formation of cold clumps/cores, but that the high (column) densities required also prohibit the destruction of molecules. Hence molecular gas is not a requirement, but form simultaneously. Therefore, the layered structure does not affect the star formation within G18.93. Instead, shielding of the radiation field is most important and the layered structure proves the effectiveness of the shielding.

\subsection{The temperature distribution}

As described in Sect. 3.3.2, the hot ionizing source as well as the IRAS source heat up their environment and produce a temperature gradient across G18.93 (cf. Fig. 5). On large scales shown by the top panel of Fig. 5, beyond the heating from the ionizing source the fitted dust temperature drops to values between $19 \mathrm{~K}$ and $20 \mathrm{~K}$, typical for the ISM (Reach et al. 1995). Within, the temperatures towards the continuum peaks stand out against their direct vicinity. However, all dense clumps are warmer than the general dust/ISM temperature.

A different visualization of the temperature gradient becomes visible if we compare the mid-IR wavelengths. Figure 9 shows a cut through G18.93/m for different wavelengths, ranging from $8 \mu \mathrm{m}$ to $870 \mu \mathrm{m}$. While the longest wavelengths trace the column density peak of the cold dust, the SED peak moves along the temperature gradient towards the heating source. Therefore, the shorter wavelengths are offset towards the heating source as well and we can trace its transition directly if we employ the full resolution at all wavelengths. Only the $8 \mu \mathrm{m}$ band is not consistent with that picture. Since it is dominated by PAH emission, its peak is influenced by the ionizing radiation.

IRDC temperatures elevated above the general ISM temperature of $\sim 18 \mathrm{~K}$ are different from typical regions of both low- and high-mass star formation (Peretto et al. 2010; Battersby et al. 2011; Nielbock et al. 2012; Launhardt et al. 2013) in which their temperatures (or the ones of dense cores) drop to temperatures below the ISM value. In contrast to the above studies, Beuther et al. (2012) find values for IRDC 18454 similar to what we find. In IRDC 18454, the mini starburst cluster W43 raises the general temperature of the IRDC complex to similar temperatures as we find for G18.93. Beuther et al. (2012) point out that the elevation of the dust temperature raises the Jeans length/mass, and therefore speculate that high-mass star formation may be favored. Hence, it could be that it is not the expansion of the HII region and the connected shock front that promotes the formation of $\mathrm{OB}$ associations on the rims of bubbles, but the effects of heating on the environment.

Therefore we conclude that the bubble does have an effect on the temperature of the IRDC, but we cannot distinguish whether this is only due to the heating source or also because of the shock front.

\subsection{Does the HII region change the shape of the IRDC?}

Elmegreen (1998) explains the formation of fingers (or elephant trunk like structures) as a shock wave runs over density enhancements and this has been directly observed (e.g. Motte et al. 2010). For the interface between G18.93 and the neighboring bubble no such structures are visible in Fig. 2 nor at other wavelengths available.

In the picture of the "collect and collapse" model the HII region pushes material along. In the vicinity of a pre-existing IRDC, the shock wave mainly penetrates one side of the IRDC, the one facing the HII region. Thus the IRDC should become asymmetric, with a steeper density profile towards the bubble. Such an asymmetry should be reflected in the projected profiles of the IRDC.

Figure 10 shows profiles of various gas tracers along two lines. While the profile through clump G18.93/1 shows a second peak along the profile and is therefore unsuited for comparing the shape of the profile, the cut through G18.93/m seems to be well suited. Figure 10 shows that ${ }^{13} \mathrm{CO}$ is more of an envelope around the entire complex and the HII peak is enclosed within the low density gas. Different from that, the dense gas peak from ATLASGAL data is clearly narrower. However, fitting both sides of the sub-mm continuum intensity distribution of G18.93/m with an exponential function independently, we find no difference between both sides of the profile. At the resolution 

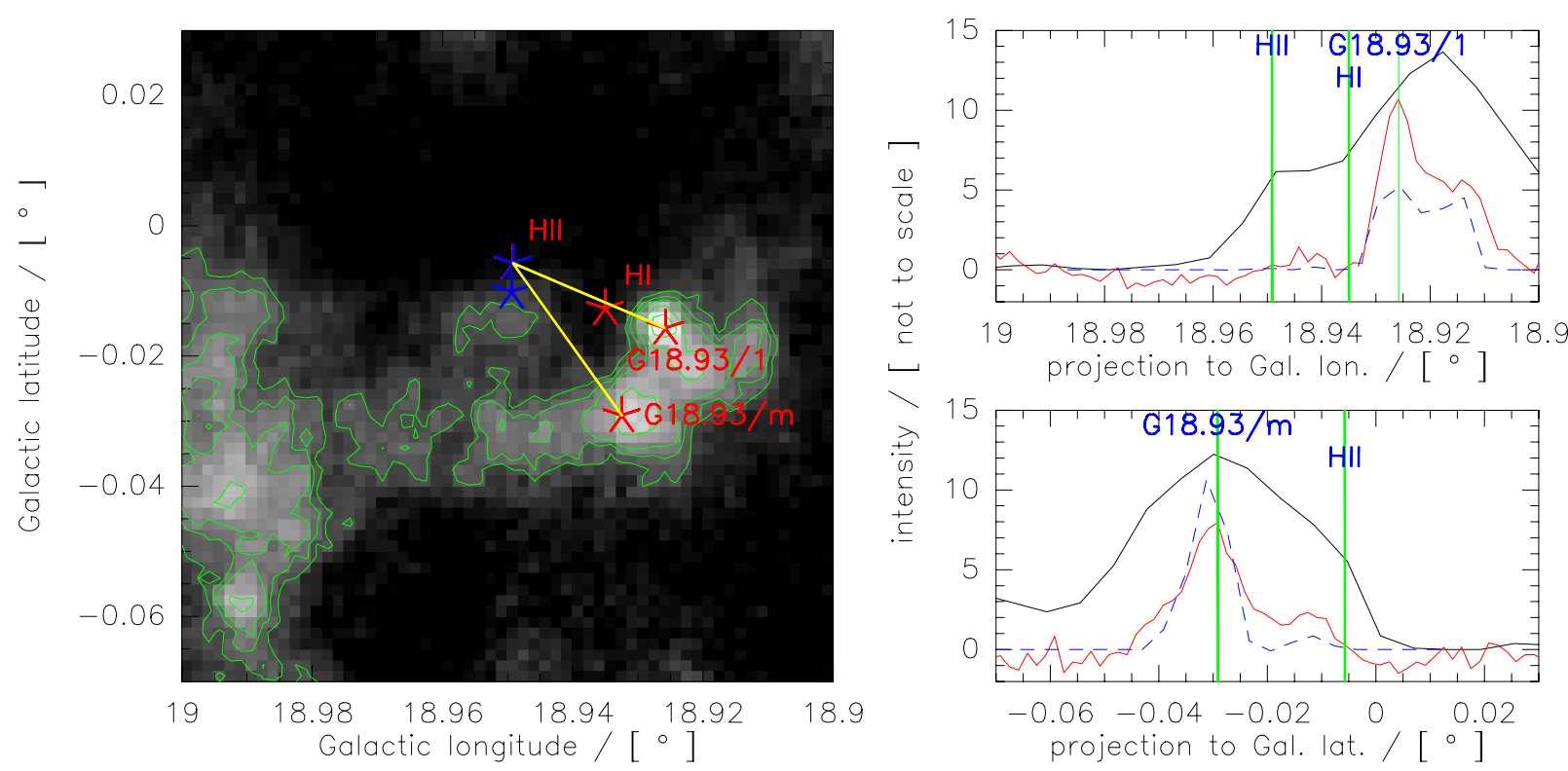

Fig. 10. Profile of ${ }^{13} \mathrm{CO}$ (black), ATLASGAL (red), and $\mathrm{H}^{13} \mathrm{CO}^{+}$(dashed blue) along the two yellow lines shown on top of the $870 \mu \mathrm{m}$ map in the left plot. The position of the peak of HII emission is indicated within both profiles (right) and the map (left). The left panel also gives the two main IRDC peaks. The blue asterisks indicate the position of the IRAS source (lower position) and UKIDSS source (higher position). The green contours from ATLASGAL are at $0.3 \mathrm{Jy}, 0.4 \mathrm{Jy}, 0.5 \mathrm{Jy}, 0.7 \mathrm{Jy}, 0.9 \mathrm{Jy}, 1.3 \mathrm{Jy}, 1.8 \mathrm{Jy}$, and $2.5 \mathrm{Jy}$.

of the IRAM $\mathrm{H}^{13} \mathrm{CO}^{+}$maps we do not have sufficient data points to do statistically meaningful fits to both slopes, but on the sparse data available, again we find no difference.

Therefore we conclude that at least for G18.93/m the bubble does not seem to influence the density profile of the IRDC.

\subsection{Imprints of the shock in the dense gas?}

After the fast (super sonic) initial expansion of an HII region to the size of a Strömgen sphere, the shock's velocity quickly drops below the sound speed $v_{\mathrm{S}}$ of the medium. Typical values of $v_{\mathrm{S}}$ are close to $10 \mathrm{~km} \mathrm{~s}^{-1}$ for the hot ionized gas. As the shock front hits the dense gas of G18.93, the expansion velocity drops dramatically, while the lower density gas towards higher latitudes allows an accelerated expansion producing the "champagne flow". However, even the slow shock front should still leave its imprint in the dense gas.

In order to search the potentially shocked gas for velocity peculiarities, we examined $\mathrm{H}^{13} \mathrm{CO}^{+}$spectra at various positions.

For three clumps, Fig. 11 compares $\mathrm{H}^{13} \mathrm{CO}^{+}$spectra within the $8 \mu \mathrm{m}$ rim to spectra at the clump's peak position and positions away from the HII region. Closest to the shock front, the spectra of G18.93/m and G18.93/1, the two right panels of Fig. 11, consist clearly of two distinct velocity components of similar strength. Since $\mathrm{H}^{13} \mathrm{CO}^{+}$is expected to be optically thin, we can exclude self absorption. The spectra at the position of the ATLASGAL continuum peaks show two components at the same velocities. However, here one component is significantly stronger than before. At the third position, away from the HII region, G18.93/m shows only a single component, while we do not detect signal for G18.93/1.

A possible interpretation of those spectra could be that one component traces the IRDC, while the second component is shock induced. In that context the rising component towards the peak of the IRDC traces the density distribution of the dense gas. Its distribution is almost symmetric perpendicular to the filament. The second component is bright towards the HII region, but due to the IRDC's high density, the shock has not propagated beyond the peak and therefore no shock component is visible away from the shock front. For the spectra taken across G18.93/4, within our sensitivity limits we cannot find similar clear imprints. Although the spectrum at its peak position has no pure Gaussian profile and a second component is required to fit it, a similar interpretation as above does not work. In Sect. 4.2, we argued that the layered structure breaks down clumps at higher longitudes, because of their lower density. For the same reason, the shock might have had less impact on the dense gas at the center of the filament. Therefore, the imprints in the spectra are not as clear.

It is interesting to note that Klessen et al. (2005) finds similar imprints on quiescent cores. For their modeling of dense cores in which the turbulence is driven on large scales, the velocity dispersion $\sigma_{\text {turb }}$ of their simulated cores show bow-shaped enhancements (rims) around the continuum emission. In our picture, instead of a large scale convergent flow, the shock introduces turbulence on large scales. The fact that Klessen et al. (2005) measure an increase of line-width instead of two separate velocity components could well be a result of their method for determining $\sigma_{\text {turb }}$.

However, the HII region does influence the dense gas of the IRDC. The additional velocity component might give the IRDC additional turbulent support to build more massive fragments.

Another possible imprint of a shock on dense gas could be a broadened line width. Within the limits of our $\mathrm{H}^{13} \mathrm{CO}^{+}$maps, we do not observe such a broadening.

\section{Conclusion}

In the middle of a $>55$ pc long filament is G18.93, a prominent IRDC. While it has the luminous protostellar object EGO G19.01-0.03 at one end, the other end is dark up to $160 \mu \mathrm{m}$. Particularly interesting about G18.93 is its environment; it is located at the projected interface of two IR bubbles. However, it is only spatially coincident with the bubble above the filament, while the bubble at lower latitudes has a different velocity. 

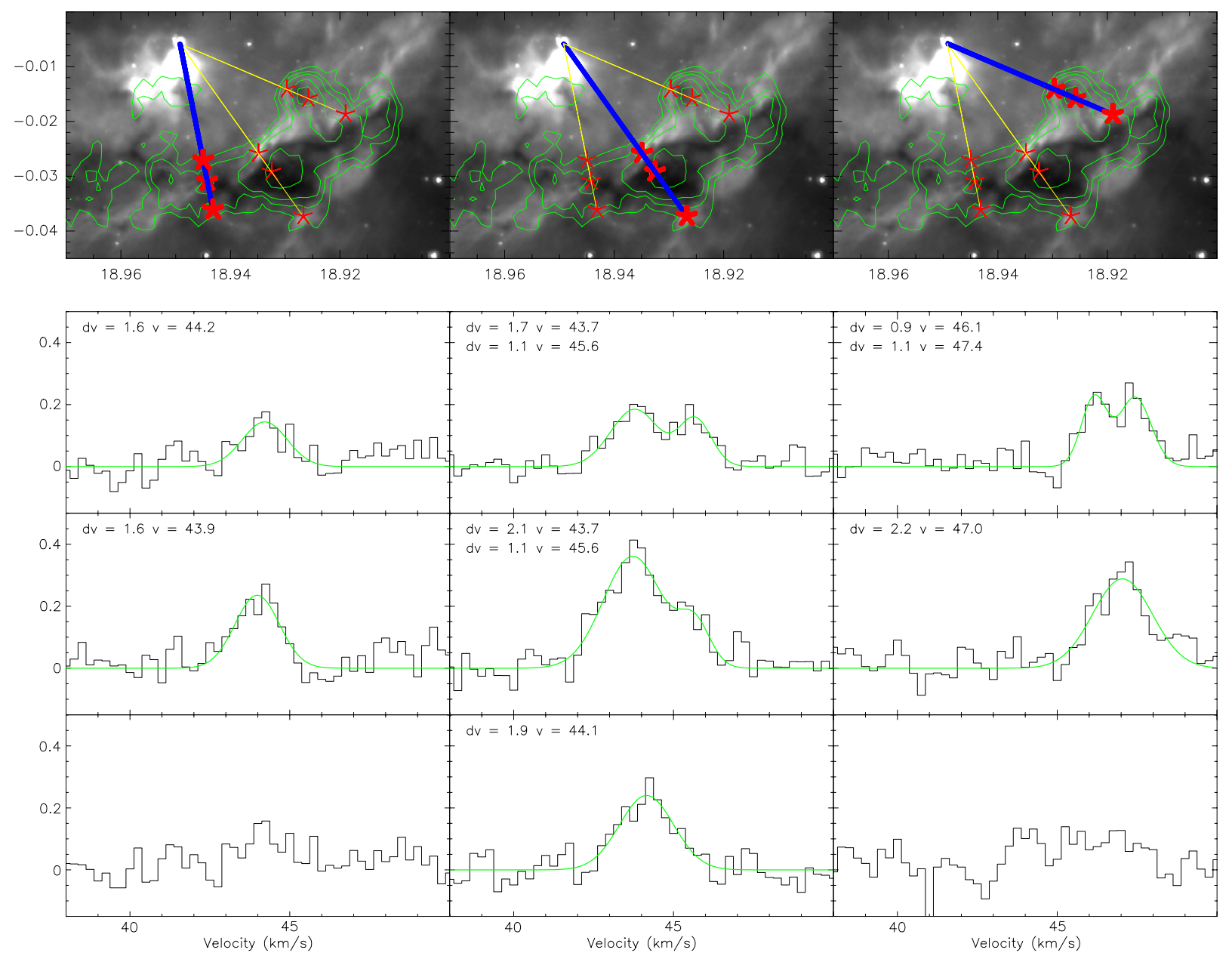

Fig. 11. $\mathrm{H}^{13} \mathrm{CO}^{+}$spectra at positions indicated by bold asterisks in the top panel. The positions are chosen to lie along the connection between the UKIDSS near-IR source and the ATLASGAL peak position, with the first spectra at the peak of the GLIMPSE $8 \mu$ m emission. From left to right the clumps are G18.93/1, G18.93/m, and G18.93/4. The upper spectra are at positions closer to the ionizing source. Where possible we fitted one, two, or three gaussian components to the spectra. The fitted line widths and peak positions are given in the spectra.

Using $\mathrm{H}^{13} \mathrm{CO}^{+}$as dense gas tracer we unambiguously attribute kinematic distances to the ATLASGAL continuum emission. Although very close in projection, the high-mass star forming region EGO G19.01-0.03 and its cold dust is not connected to G18.93, but exists at a different distance. From a Galactic rotation curve we determined IRDC G18.93's distance to be $3.6 \mathrm{kpc}$.

We used CLUMPFIND to decompose the dense gas seen by ATLASGAL into 6 clumps. Together with temperature estimates from HiGal SED fitting, we calculated both the column density and masses of these clumps. The most massive clump is G18.93/m with a gas mass of $\sim 280 M_{\odot}$. A comparison of the gas mass to its virial mass of $360 M_{\odot}$ shows that within the uncertainties it could be gravitationally bound. To determine its evolutionary stage, we first searched the GLIMPSE catalog for young stellar objects using IRAC color criteria, but no young stellar object was found within the boundaries of G18.93/m. Next we visually inspected the longer wavelength images MIPSGAL $24 \mu \mathrm{m}$ and HiGal and find no point source at wavelength up to $160 \mu \mathrm{m}$.

In addition, the absence of $\mathrm{SiO}$ emission is strongly indicative of no star formation activity. Therefore, we identify G18.93/m as a potential high-mass pre-stellar clump.

Expanding the SED fitting from single positions to all pixels, we produced temperature maps. The sources embedded in the dust connected to IRAS18227-1227 heat the dust and produce a strong gradient across the IRDC. Therefore, all IR dark continuum peaks have temperatures above the general ISM value of $\sim 19 \mathrm{~K}$.

In context of the bubble we show that the "champagne flow" structure is produced by an expanding HII region. From measuring the radio continuum flux we estimate the number of Lyman continuum photons and constrain the ionizing source to be at least an O8.5 star.

From VGPS HI spectra we extract a measure of the HI column density. Together with the $\mathrm{cm}$ continuum data and the ATLASGAL data we identify a layered structure between the ionizing source and the IRDC, from ionized through atomic to molecular hydrogen.

Finally, we discuss the imprints of the expanding HII region on the starless clump G18.93/m. Our main results are:

- layered structure: the ionizing source produces a layered structure towards the IRDC and therefore changes the composition of the hydrogen phase.

- temperature distribution: the IRAS source and/or the ionizing source produce a strong temperature gradient across the IRDC. Although the dust temperature at the continuum peaks is reduced compared to their surrounding, their absolute temperatures are above the ISM values. This is atypical compared to most other IRDCs. However, as discussed in 
Beuther et al. (2012), the additional support raises the Jeans length which might favor high-mass star formation.

- density profiles: in the picture of the "collapse and collect" scenario we would expect the shock front to steepen the density profile of the dense gas. Therefore we compare the emission profile of the cold gas towards and away from G18.93/m. Fitting both wings, we find no difference.

- imprints within the dense gas: looking at $\mathrm{H}^{13} \mathrm{CO}^{+}$spectra across the filament, we find emission that resembles the brightness of the ATLASGAL dust continuum. In addition we find a second $\mathrm{H}^{13} \mathrm{CO}^{+}$component, brightest within a PAH rim towards the bubble and not detected away from the bubble. We speculate that this might be a direct imprint of the shock front onto the IRDC.

Therefore, while the additional heating and the shock induced velocity component favor high-mass star formation, we do not find evidence for collapse triggered by the expanding HII region.

Acknowledgements. This publication is partially based on data acquired with the Atacama Pathfinder Experiment (APEX). APEX is a collaboration between the Max-Planck-Institut für Radioastronomie, the European Southern Observatory, and the Onsala Space Observatory. This publication makes use of molecular line data from the Boston University-FCRAO Galactic Ring Survey (GRS). The GRS is a joint project of Boston University and Five College Radio Astronomy Observatory, funded by the National Science Foundation under grants AST9800334, AST-0098562, \& AST-0100793. The International Galactic Plane Survey is supported through a Collaborative Research Opportunities grant from the Natural Sciences and Engineering Research Council of Canada. The National Radio Astronomy Observatory is a facility of the National Science Foundation operated under cooperative agreement by Associated Universities, Inc. This work is based, in part, on observations made with the Spitzer Space Telescope, which is operated by the Jet Propulsion Laboratory, California Institute of Technology under a contract with NASA. This research has made use of the NASA/ IPAC Infrared Science Archive, which is operated by the Jet Propulsion Laboratory, California Institute of Technology, under contract with the National Aeronautics and Space Administration. The UKIDSS project is defined in Lawrence et al. (2007). UKIDSS uses the UKIRT Wide Field Camera (WFCAM; Casali et al. 2007) and a photometric system described in Hewett et al. (2006). The pipeline processing and science archive are described in Irwin et al. (2008) and Hambly et al. (2008). We have used data from the 7th data release. J.T. is supported by the International Max Planck Research School (IMPRS) for Astronomy and Cosmic Physics.

\section{References}

Bally, J., Aguirre, J., Battersby, C., et al. 2010, ApJ, 721, 137 Battersby, C., Bally, J., Ginsburg, A., et al. 2011, A\&A, 535, A128 Benjamin, R. A., Churchwell, E., Babler, B. L., et al. 2003, PASP, 115, 953 Beuther, H., Schilke, P., Menten, K. M., et al. 2002, ApJ, 566, 945 Beuther, H., Henning, T., Linz, H., et al. 2010, A\&A, 518, L78 Beuther, H., Tackenberg, J., Linz, H., et al. 2012, A\&A, 538, A11 Bieging, J. H., Peters, W. L., Vila Vilaro, B., Schlottman, K., \& Kulesa, C. 2009, AJ, 138, 975

Blitz, L., Fich, M., \& Stark, A. A. 1982, ApJS, 49, 183

Carey, S. J., Noriega-Crespo, A., Mizuno, D. R., et al. 2009, PASP, 121, 76

Churchwell, E., Povich, M. S., Allen, D., et al. 2006, ApJ, 649, 759 Churchwell, E., Watson, D. F., Povich, M. S., et al. 2007, ApJ, 670, 428

Condon, J. J., Cotton, W. D., Greisen, E. W., et al. 1998, AJ, 115, 1693

Cyganowski, C. J., Whitney, B. A., Holden, E., et al. 2008, AJ, 136, 2391

Cyganowski, C. J., Brogan, C. L., Hunter, T. R., \& Churchwell, E. 2011a, ApJ, 743,56

Cyganowski, C. J., Brogan, C. L., Hunter, T. R., Churchwell, E., \& Zhang, Q. 2011b, ApJ, 729, 124

Deharveng, L., Lefloch, B., Zavagno, A., et al. 2003, A\&A, 408, L25

Deharveng, L., Schuller, F., Anderson, L. D., et al. 2010, A\&A, 523, A6

Elmegreen, B. G. 1998, in Origins, eds. C. E. Woodward, J. M. Shull, \& H. A. Thronson, Jr., ASP Conf. Ser., 148, 150
Elmegreen, B. G., \& Lada, C. J. 1977, ApJ, 214, 725

Fich, M., Treffers, R. R., \& Blitz, L. 1982, in Regions of Recent Star Formation, eds. R. S. Roger, \& P. E. Dewdney, Ap\&SS Libr., 93, 201

Glover, S. C. O., \& Clark, P. C. 2012, MNRAS, 421, 9

Gutermuth, R. A., Myers, P. C., Megeath, S. T., et al. 2008, ApJ, 674, 336

Hatchell, J., \& van der Tak, F. F. S. 2003, A\&A, 409, 589

Helfand, D. J., Becker, R. H., White, R. L., Fallon, A., \& Tuttle, S. 2006, AJ, 131,2525

Henning, T., Linz, H., Krause, O., et al. 2010, A\&A, 518, L95

Hoare, M. G., Purcell, C. R., Churchwell, E. B., et al. 2012, PASP, 124, 939

Hollenbach, D. J., \& Tielens, A. G. G. M. 1997, ARA\&A, 35, 179

Jackson, J. M., Rathborne, J. M., Shah, R. Y., et al. 2006, ApJS, 163, 145

Jackson, J. M., Finn, S. C., Chambers, E. T., Rathborne, J. M., \& Simon, R. 2010, ApJ, 719, L185

Kainulainen, J., Alves, J., Beuther, H., Henning, T., \& Schuller, F. 2011, A\&A, 536, A48

Kendrew, S., Simpson, R. J., Bressert, E., et al. 2012, ApJ, 755, 71

Klessen, R. S., Ballesteros-Paredes, J., Vázquez-Semadeni, E., \& Durán-Rojas, C. 2005, ApJ, 620, 786

Krumholz, M. R., \& McKee, C. F. 2008, Nature, 451, 1082

Kuchar, T. A., \& Clark, F. O. 1997, ApJ, 488, 224

Kurtz, S., Churchwell, E., \& Wood, D. O. S. 1994, ApJS, 91, 659

Launhardt, R., Stutz, A. M., Schmiedeke, A., et al. 2013, A\&A, in press, DOI: 10.1051/0004-6361/201220477

Lawrence, A., Warren, S. J., Almaini, O., et al. 2007, MNRAS, 379, 1599

Li, D., \& Goldsmith, P. F. 2003, ApJ, 585, 823

Lockman, F. J. 1989, ApJS, 71, 469

MacLaren, I., Richardson, K. M., \& Wolfendale, A. W. 1988, ApJ, 333, 821

Martins, F., Schaerer, D., \& Hillier, D. J. 2005, A\&A, 436, 1049

Mezger, P. G., \& Henderson, A. P. 1967, ApJ, 147, 471

Molinari, S., Swinyard, B., Bally, J., et al. 2010, PASP, 122, 314

Motte, F., Bontemps, S., Schilke, P., et al. 2007, A\&A, 476, 1243

Motte, F., Zavagno, A., Bontemps, S., et al. 2010, A\&A, 518, L77

Nielbock, M., Launhardt, R., Steinacker, J., et al. 2012, A\&A, 547, A11

Ossenkopf, V., \& Henning, T. 1994, A\&A, 291, 943

Ott, S. 2010, in Astronomical Data Analysis Software and Systems XIX, eds. Y. Mizumoto, K.-I. Morita, \& M. Ohishi, ASP Conf. Ser., 434, 139 Panagia, N. 1973, AJ, 78, 929

Parker, Q. A., Phillipps, S., Pierce, M. J., et al. 2005, MNRAS, 362, 689

Peretto, N., \& Fuller, G. A. 2009, A\&A, 505, 405

Peretto, N., Fuller, G. A., Plume, R., et al. 2010, A\&A, 518, L98

Pillai, T., Wyrowski, F., Carey, S. J., \& Menten, K. M. 2006, A\&A, 450, 569

Purcell, C. R., \& Hoare, M. G. 2010, Highlights of Astronomy, 15, 781

Purcell, C. R., Hoare, M. G., \& Diamond, P. 2008, in Massive Star Formation: Observations Confront Theory, eds. H. Beuther, H. Linz, \& T. Henning, ASP Conf. Ser., 387, 389

Ragan, S., Henning, T., Krause, O., et al. 2012, A\&A, 547, A49

Rathborne, J. M., Jackson, J. M., \& Simon, R. 2006, ApJ, 641, 389

Reach, W. T., Dwek, E., Fixsen, D. J., et al. 1995, ApJ, 451, 188

Reich, W., Reich, P., \& Fuerst, E. 1990, A\&AS, 83, 539

Reid, M. J., Menten, K. M., Zheng, X. W., et al. 2009, ApJ, 700, 137

Robitaille, T. P., Meade, M. R., Babler, B. L., et al. 2008, AJ, 136, 2413

Rosolowsky, E., Dunham, M. K., Ginsburg, A., et al. 2010, ApJS, 188, 123

Roussel, H. 2012 [arXiv: 1205.2576]

Russeil, D., Zavagno, A., Motte, F., et al. 2010, A\&A, 515, A55

Schilke, P., Walmsley, C. M., Pineau des Forets, G., \& Flower, D. R. 1997, A\&A, 321,293

Schuller, F., Menten, K. M., Contreras, Y., et al. 2009, A\&A, 504, 415

Simon, R., Jackson, J. M., Rathborne, J. M., \& Chambers, E. T. 2006, ApJ, 639, 227

Simpson, R. J., Povich, M. S., Kendrew, S., et al. 2012, MNRAS, 424, 2442

Siringo, G., Kreysa, E., Kovács, A., et al. 2009, A\&A, 497, 945

Stahler, S. W., \& Palla, F. 2005, The Formation of Stars

Stil, J. M., Taylor, A. R., Dickey, J. M., et al. 2006, AJ, 132, 1158

Stutz, A., Launhardt, R., Linz, H., et al. 2010, A\&A, 518, L87

Tackenberg, J., Beuther, H., Henning, T., et al. 2012, A\&A, 540, A113

Traficante, A., Calzoletti, L., Veneziani, M., et al. 2011, MNRAS, 416, 2932

Vasyunina, T., Linz, H., Henning, T., et al. 2009, A\&A, 499, 149

Vasyunina, T., Linz, H., Henning, T., et al. 2011, A\&A, 527, A88

Wienen, M., Wyrowski, F., Schuller, F., et al. 2012, A\&A, 544, A146

Williams, J. P., de Geus, E. J., \& Blitz, L. 1994, ApJ, 428, 693

Zavagno, A., Deharveng, L., Comerón, F., et al. 2006, A\&A, 446, 171

Zavagno, A., Anderson, L. D., Russeil, D., et al. 2010, A\&A, 518, L101 